\title{
Relativistic bound-state equations in three dimensions
}

\author{
D. R. Phillips and S. J. Wallace * \\ Department of Physics and Center for Theoretical Physics, \\ University of Maryland, College Park, MD, 20742-4111
}

\begin{abstract}
Firstly, a systematic procedure is derived for obtaining three-dimensional bound-state equations from four-dimensional ones. Unlike "quasi-potential approaches" this procedure does not involve the use of delta-function constraints on the relative four-momentum. In the absence of negative-energy states, the kernels of the three-dimensional equations derived by this technique may be represented as sums of time-ordered perturbation theory diagrams. Consequently, such equations have two major advantages over quasi-potential equations: they may easily be written down in any Lorentz frame, and they include the meson-retardation effects present in the original four-dimensional equation. Secondly, a simple four-dimensional equation with the correct onebody limit is obtained by a reorganization of the generalized ladder BetheSalpeter kernel. Thirdly, our approach to deriving three-dimensional equations is applied to this four-dimensional equation, thus yielding a retarded interaction for use in the three-dimensional bound-state equation of Wallace and Mandelzweig. The resulting three-dimensional equation has the correct one-body limit and may be systematically improved upon. The quality of the three-dimensional equation, and our general technique for deriving such equations, is then tested by calculating bound-state properties in a scalar field theory using six different bound-state equations. It is found that equations obtained using the method espoused here approximate the wave functions obtained from their parent four-dimensional equations significantly better than the corresponding quasi-potential equations do.
\end{abstract}

\section{INTRODUCTION}

With the advent of experimental facilities such as CEBAF, which are capable of probing hadronic systems at energies where relativistic effects become important, the development of theoretical frameworks in which consistent relativistic calculations of few-body hadronic systems can be performed is no longer merely desirable, it is essential.

\footnotetext{
*Email: phillips@quark.umd.edu,wallace@quark.umd.edu.
} 
An obvious starting point for such a calculation in a two-body system (such as the deuteron) is the Bethe-Salpeter equation (BSE) for the four-dimensional, covariant, two-totwo amplitude $T$,

$$
T=K+K G_{0} T
$$

where $G_{0}$ is the free two-particle propagator, which in our convention is

$$
G_{0}\left(p_{1}^{\prime}, p_{2}^{\prime} ; p_{1}, p_{2}\right)=i(2 \pi)^{8} \delta^{(4)}\left(p_{1}^{\prime}-p_{1}\right) \delta^{(4)}\left(p_{2}^{\prime}-p_{2}\right) d_{1}\left(p_{1}\right) d_{2}\left(p_{2}\right)
$$

with

$$
d_{i}\left(p_{i}\right)=\frac{\Lambda_{i}^{+}\left(\mathbf{p}_{i}\right)}{p_{i}^{0}-\epsilon_{i}\left(\mathbf{p}_{i}\right)+i \eta}-\frac{\Lambda_{i}^{-}\left(\mathbf{p}_{i}\right)}{p_{i}^{0}+\epsilon_{i}\left(\mathbf{p}_{i}\right)-i \eta}
$$

where

$$
\begin{aligned}
\epsilon_{i}\left(\mathbf{p}_{i}\right) & =\sqrt{\mathbf{p}_{i}^{2}+m_{i}^{2}}, \\
\Lambda_{i}^{ \pm}\left(\mathbf{p}_{i}\right) & = \begin{cases}\frac{1}{2 \epsilon_{i}\left(\mathbf{p}_{i}\right)}, & \text { for spin-zero particles, } \\
\frac{ \pm \epsilon_{i}\left(\mathbf{p}_{i}\right) \gamma^{0}-\gamma_{i} \cdot \mathbf{p}_{i}+m_{i}}{2 \epsilon_{i}\left(\mathbf{p}_{i}\right)}, & \text { for spin-half particles }\end{cases}
\end{aligned}
$$

$\eta$ is a positive infinitessimal and $K$ is the Bethe-Salpeter kernel [1] [5]. In principle $K$ should include all two-particle irreducible two-to-two Feynman graphs. Solution of (1.1) with the full two-particle irreducible kernel is impractical and usually resort is made to the ladder approximation [6-9]. Some undesirable features attend this approximation (see [10] for a full discussion), not the least of which is the fact that the ladder BSE does not have the correct one-body limit [11]. (An equation is said to have the correct one-body limit if, when the mass of one particle is taken to infinity, the equation reduces to the Klein-Gordon or Dirac equation for the light particle moving in the static field of the now infinitely massive source.) By contrast, the full BSE (1.1) does possess the correct one-body limit.

Three-dimensional quasi-potential equations (QPEs) are realized by rewriting the BetheSalpeter equation for the two-particle amplitude (1.1) as a pair of coupled equations:

$$
\begin{aligned}
& T=U+U g T, \\
& U=K+K\left(G_{0}-g\right) U,
\end{aligned}
$$

where the propagator $g$ is arbitrary. If $g$ is chosen to contain a one-dimensional delta function constraining the relative four-momentum, then Eq. (1.6) becomes a three-dimensional equation for the amplitude $T$. Examples of this approach include the equations of BlankenbeclerSugar [12] and Gross [11,13]. In principle the driving term $U$ should be determined by solving the four-dimensional integral equation (1.7). However, given the difficulty of solving such equations, and our lack of knowledge about the best form of $K$ for hadronic physics, usually $U$ is chosen to be a one-boson exchange interaction:

$$
U=V_{O B E}
$$

The t-matrix then obeys the three-dimensional integral equation 


$$
T=V_{O B E}+V_{O B E} g T .
$$

A reasonable description of few-hadron systems is obtained by fitting coupling constants and some mass parameters of $V_{O B E}$ to the nucleon-nucleon scattering data. (Examples of this approach include, but are by no means limited to, Refs. [14 [16].)

The QPE formalism of Gross obeys the one-body limit [11]. Another variant of the quasi-potential approach which respects the one-body limit was derived by Wallace and Mandelzweig in References [17 19]. We provide a generalization of that formalism in this paper. As originally derived, the QPE of Wallace and Mandelzweig contains, for the nucleonnucleon system, a one-boson exchange potential which is instant in the center-of-mass frame. This involves the use of a constraint on the relative four-momentum, $p$, of the form

$$
p \cdot P=0,
$$

where $P$ is the total two-body four-momentum. In the center-of-mass frame, the equation for the deuteron vertex function takes the form:

$$
\Gamma_{W M}=V_{\mathrm{inst}} G_{E T} \Gamma_{W M},
$$

where $G_{E T}$ refers to the Wallace-Mandelzweig choice for the propagator $g$. The formalism is covariant since the constraint (1.10) is expressed covariantly and the pair of equations (1.11) and (1.7) is equivalent to the bound-state BSE:

$$
\Gamma=K G_{0} \Gamma
$$

Eq. (1.11) for the $\mathrm{cm}$ frame deuteron vertex function has been used in recent work by Devine and Wallace [20,21]. This formalism may be developed in a manifestly covariant fashion following the technique of Fuda [22]. This involves writing all four-vectors in terms of their components parallel and perpendicular to the total four-momentum:

$$
p=p_{\|} \hat{P}+p_{\perp}
$$

where $p_{\|}=p \cdot \hat{P}$, with $\hat{P}=P / \sqrt{P^{2}}$.

A fundamental flaw exists in quasipotential formalisms that are based on a form for $g$ which contains a delta function. It is generally impossible to systematically correct the lowest-order approximation by use of Eq. (1.7) because unphysical singularities arise. In two-body hadronic systems, such as the deuteron, calculations may still be pursued using equations such as (1.11) which are themselves free of these singularities. On the other hand, in any electromagnetic reaction where the photon momentum $q$ is non-zero, the constraint (11.10) cannot hold for the initial and final-state relative four-momenta in the cm frame [20]. Therefore a boosted vertex function is required for electromagnetic matrix element calculations, or, for that matter, for any QPE calculation in a three-body problem. A boost equation for the quasipotential may be deduced from Eq. (1.7), but unphysical singularities arise in the boosted interaction [20,21]. These are removed from the theory if the full result for $U$, as defined by (1.7), is used in the quasi-potential equation, but no truncation of $U$ at any finite order in $G-g$ is free of singularities.

Therefore, in this paper we seek a general procedure for the reduction of four-dimensional equations which does not involve the use of delta functions. We develop a procedure by 
which a four-dimensional equation may be approximated by a three-dimensional equation and the interaction in that equation improved systematically. The technique is presented in Section [1]. It has a close connection to the work of Klein [23 26] on three-dimensional reductions of four-dimensional equations, and to standard time-ordered perturbation theory, as discussed in Section III.

In general the interaction in the three-dimensional equations we discuss is not covariant. But, since the application of our delta-function-free reduction technique to infinite order produces an equation equivalent to the original four-dimensional equation, the sum of all terms in the three-dimensional formalism must produce covariant results. In nuclear physics it is known that contributions which are of higher-order in the coupling are of increasingly shorter range. Such short-range contributions to hadronic interactions must always be treated in an essentially phenomenological manner. Therefore, it is expected that a truncation of the interaction at some finite order in the coupling will be useful for applications. It should always be appropriate to absorb the effects of the neglected higher-order graphs into phenomenological parameters, thus ameliorating the non-covariance of the theory. In particular, we show in Section III that the leading-order boost corrections to the interaction obtained by Forest, Pandharipande and Friar [27] are indeed contained within our truncated interaction.

In Section $\mathbb{I V}$ we show that the crossed-box graph may be approximately rewritten as an iterate of the ladder kernel:

$$
K_{X}^{(4)} \approx K^{(2)} G_{C} K^{(2)}
$$

where the form of $G_{C}$ is derived in Section IV. In particular, this approximation is exact in the high-energy (eikonal) and one-body limits, and may be systematically improved upon in other regimes. This leads us to propose the four-dimensional integral equation:

$$
\Gamma=K^{(2)}\left(G_{0}+G_{C}\right) \Gamma .
$$

This equation has the correct one-body limit and results from an approximate resummation of the BSE kernel $K$. It reduces to the Wallace-Mandelzweig equation (1.11) if the dependence of $K^{(2)}$ on the time component of the relative four-momentum in the cm frame is neglected. A three-dimensional reduction of Eq. (1.15) using the method developed in Section 11 provides a systematic way to include retardation effects, and so improve on Eq. (1.11). This reduction to a three-dimensional equation is performed in Section $\mathrm{V}$. The dynamical boost of the three-dimensional interaction present in the equation is realized through simple dependence of the interaction on the total three momentum and energy of the system, thus eliminating the boost problem of Eq. (1.11).

In Section VI the predictions of the three-dimensional integral equation obtained in Section $\nabla$ are compared to those of five other bound-state equations: the ladder BSE and Eq. (1.15), both of which are, of course, four-dimensional equations, and the threedimensional equations of Salpeter, Klein and Wallace \& Mandelzweig.

The results of Sections III- $\square$ are presented concurrently for a scalar and a spinor field theory. In particular, in the scalar case we use the $\phi^{2} \sigma$ field theory, defined by the Lagrangian

$$
\mathcal{L}=\frac{1}{2}\left(\partial_{\mu} \phi_{1} \partial^{\mu} \phi_{1}-m_{1}^{2} \phi_{1}^{2}+\partial_{\mu} \phi_{2} \partial^{\mu} \phi_{2}-m_{2}^{2} \phi_{2}^{2}+\partial_{\mu} \sigma \partial^{\mu} \sigma-\mu^{2} \sigma^{2}\right)-g_{1} m_{1} \phi_{1}^{2} \sigma-g_{2} m_{2} \phi_{2}^{2} \sigma .
$$


Coupling terms include mass factors so that the couplings $g_{1}$ and $g_{2}$ are dimensionless, thus ensuring that the limits $m_{1} \rightarrow \infty$ and $m_{2} \rightarrow \infty$ correspond to the appropriate one-body limits. It is this field theory in which the numerical calculations of Section $\nabla \mathbb{1}$ are performed. In the spinor case the Lagrangian is

$$
\mathcal{L}=\bar{\psi}_{1}\left(i \gamma_{\mu} \partial^{\mu}-m_{1}\right) \psi_{1}+\bar{\psi}_{2}\left(i \gamma_{\mu} \partial^{\mu}-m_{2}\right) \psi_{2}-g_{1} \bar{\psi}_{1} \sigma \psi_{1}-g_{2} \bar{\psi}_{2} \sigma \psi_{2}
$$

The arguments of Sections III $\square$ are, in fact, quite general, and, with small modifications, also apply to field theories involving other types of particles.

\section{A SYSTEMATIC PROCEDURE FOR THE REMOVAL OF THE RELATIVE-ENERGY DEGREE OF FREEDOM FROM A BOUND-STATE EQUATION}

Consider the Bethe-Salpeter equation for the bound-state vertex function $\Gamma$, i.e.,

$$
\Gamma(p ; P)=\int \frac{d^{4} p^{\prime}}{(2 \pi)^{4}} K\left(p, p^{\prime} ; P\right) G_{0}\left(p^{\prime} ; P\right) \Gamma\left(p^{\prime} ; P\right),
$$

Here $P$ is the total two-body four-momentum, and $p$ and $p^{\prime}$ are the relative four-momenta. This equation is completely general, and applies to the bound-state vertex function in any field theory. Throughout this paper we find it convenient to omit the explicit integration from such equations, abbreviating them as follows

$$
\Gamma=K G_{0} \Gamma
$$

Suppose that the driving term of this equation, $K$, is separated into two pieces:

$$
K=K_{1}+K_{2},
$$

where $K_{1}$ is three-dimensional in the sense that it does not depend on the zeroth component of the relative four-momenta $p$ and $p^{\prime}$. For instance, if (2.1) was being solved in the two-body center-of-mass frame and $K$ was a one-boson-exchange kernel then $K_{1}$ could be chosen to be the usual static one-boson-exchange kernel. Note that even though the whole driving term $K$ is Lorentz covariant, because the restriction on $K_{1}$ is frame dependent, the pieces $K_{1}$ and $K_{2}$ are not. The splitting of $K$ leads to the following coupled equations for $\Gamma$ :

$$
\begin{aligned}
\Gamma & =\Gamma_{1}+\Gamma_{2}, \\
\Gamma_{1} & =K_{1} G_{0}\left(\Gamma_{1}+\Gamma_{2}\right), \\
\Gamma_{2} & =K_{2} G_{0}\left(\Gamma_{1}+\Gamma_{2}\right) .
\end{aligned}
$$

Both these equations are four-dimensional, however, $\Gamma_{1}$ has no dependence on the zeroth component of the relative four-momentum because of the defining condition of $K_{1}$. Meanwhile, the second equation may be formally solved to obtain:

$$
\begin{aligned}
\Gamma_{2} & =\left[1-K_{2} G_{0}\right]^{-1} K_{2} G_{0} \Gamma_{1} \\
& =-\Gamma_{1}+\left[1-K_{2} G_{0}\right]^{-1} \Gamma_{1} .
\end{aligned}
$$


This last result may then be substituted into Eq. (2.5) to yield

$$
\Gamma_{1}=K_{1} \mathcal{G} \Gamma_{1},
$$

where

$$
\mathcal{G}=G_{0}+G_{0} K_{2} \mathcal{G}
$$

Eq. (2.8) then becomes a three-dimensional equation because the implied integrations over time-components of momenta only affect $\mathcal{G}$, i.e., it reduces to

$$
\Gamma_{1}=K_{1}\langle\mathcal{G}\rangle \Gamma_{1},
$$

where

$$
\langle\mathcal{G}\rangle \equiv \int \frac{d p_{0}^{\prime} d p_{0}}{(2 \pi)^{2}} \mathcal{G}\left(p_{\mu}^{\prime}, p_{\mu} ; P\right)
$$

Here we have used the same implied integration notation for the three-dimensional integral equation (2.10) as for a four-dimensional equation. This practice continues below and the context should make it clear whether the equation in question is a three or four-dimensional one.

Eqs. 2.10) and (2.9) are exactly equivalent to the original BSE (2.1). In order to reconstruct the Bethe-Salpeter amplitude $\Gamma$ from the non-covariant three-dimensional $\Gamma_{1}$ one must use Eq.(2.7) rearranged into the form

$$
\Gamma=\left[1-K_{2} G_{0}\right]^{-1} \Gamma_{1}
$$

The Green's function, $\langle\mathcal{G}\rangle$, defined by Eq. (2.11) is, in fact, the Fourier transform of the corresponding equal-time co-ordinate space Green's function $\mathcal{G}\left(t^{\prime}, \mathbf{x}_{1}^{\prime}, \mathbf{x}_{2}^{\prime} ; t, \mathbf{x}_{1}, \mathbf{x}_{2}\right)$. This may be seen by defining

$$
\mathcal{G}\left(t^{\prime}, \mathbf{x}_{1}^{\prime}, \mathbf{x}_{2}^{\prime} ; t, \mathbf{x}_{1}, \mathbf{x}_{2}\right)=\delta\left(x_{1}^{0 \prime}-x_{2}^{0 \prime}\right) \delta\left(x_{1}^{0}-x_{2}^{0}\right) g\left(x_{1}^{\prime}, x_{2}^{\prime} ; x_{1}, x_{2}\right),
$$

where $g$ is the appropriate four-dimensional two-body Green's function, then inserting the integral representation of the delta functions and taking the Fourier transform [28,29].

If $K_{1}$ is now chosen to be a cm-frame instant interaction, $K^{\text {inst }}$, then (2.9) may be taken to define $\mathcal{G}$ order by order in $K-K^{\text {inst }}$. This provides a systematic way to calculate retardation corrections to the Salpeter equation [30]

$$
\Gamma_{1}=K^{\text {inst }}\left\langle G_{0}\right\rangle \Gamma_{1}
$$

which results from taking $\mathcal{G}$ at zeroth order in $K-K^{\text {inst }}$. In particular, at first order in $K-K^{\text {inst }}$ we find that:

$$
\left\langle\mathcal{G}^{(1)}\right\rangle=\left\langle G_{0}\right\rangle+\left\langle G_{0} K G_{0}\right\rangle-\left\langle G_{0}\right\rangle K^{\text {inst }}\left\langle G_{0}\right\rangle
$$

The use of this three-dimensional propagator in place of $\left\langle G_{0}\right\rangle$ in 2.10 ) would therefore incorporate leading-order retardation corrections in the three-dimensional equation. 
There is, however, a general way to account for all the relative-energy integrations in the modified Green's function $\langle\mathcal{G}\rangle$. Recall that the choice of $K_{1}$ is subject only to the constraint that it should not depend on the zeroth component of the relative four-momentum in, say, the $\mathrm{cm}$ frame. Consider Eq. (2.9) rewritten as

$$
\langle\mathcal{G}\rangle=\left\langle G_{0}\right\rangle+\left\langle G_{0} K \mathcal{G}\right\rangle-\left\langle G_{0}\right\rangle K_{1}\langle\mathcal{G}\rangle,
$$

and choose $K_{1}$ such that $\langle\mathcal{G}\rangle=\left\langle G_{0}\right\rangle$. This provides the defining condition,

$$
K_{1} \equiv\left\langle G_{0}\right\rangle^{-1}\left\langle G_{0} K \mathcal{G}\right\rangle\left\langle G_{0}\right\rangle^{-1} .
$$

Since this choice of $K_{1}$ means that $\langle\mathcal{G}\rangle=\left\langle G_{0}\right\rangle$ the full dynamics reduces to a threedimensional integral equation with the free propagator $\left\langle G_{0}\right\rangle$, i.e.,

$$
\Gamma_{1}=K_{1}\left\langle G_{0}\right\rangle \Gamma_{1},
$$

and all the complexities of the relative-energy integrations are transferred to the interaction, $K_{1}$.

It follows that $K_{1}$ is the two-particle irreducible (2PI) interaction, where two-particle irreducibility is defined with respect to the three-dimensional propagator $\left\langle G_{0}\right\rangle$. To show this formally, rearrange $\left\langle G_{0} K \mathcal{G}\right\rangle$ as follows,

$$
\begin{aligned}
\left\langle G_{0} K \mathcal{G}\right\rangle & =\left\langle G_{0} K G_{0}\left(1-K G_{0}\right)^{-1}\right\rangle+\left\langle G_{0} K G_{0}\left\{\left[1-\left(K-K_{1}\right) G_{0}\right]^{-1}-\left[1-K G_{0}\right]^{-1}\right\}\right\rangle \\
& =\left\langle G_{0} T G_{0}\right\rangle-\left\langle G_{0} T G_{0}\right\rangle K_{1}\langle\mathcal{G}\rangle,
\end{aligned}
$$

where $T=K\left(1-G_{0} K\right)^{-1}$ is the Bethe-Salpeter t-matrix defined by Eq. (1.1). Use of Eq. (2.19) in Eq. (2.17) produces

$$
T_{1}=K_{1}+T_{1}\left\langle G_{0}\right\rangle K_{1},
$$

where $T_{1}=\left\langle G_{0}\right\rangle^{-1}\left\langle G_{0} T G_{0}\right\rangle\left\langle G_{0}\right\rangle^{-1}$ is the three-dimensional t-matrix corresponding to the Bethe-Salpeter t-matrix $T$. Thus $K_{1}$ is, indeed, the irreducible interaction which produces the t-matrix $T_{1}$ using the propagator $\left\langle G_{0}\right\rangle$. We therefore expect that the rules for the construction of $K_{1}$ will be akin to those for the two-particle irreducible interaction in timeordered perturbation theory.

\section{CONNECTION TO TIME-ORDERED PERTURBATION THEORY AND THE WORK OF KLEIN}

The connection to time-ordered perturbation theory (TOPT) emerges for a simplified dynamics in which only positive-energy states are kept in $G_{0}$. For the case of a field theory of nucleons and pions it has been shown that the Green's function $\left\langle G_{0}+G_{0} T G_{0}\right\rangle$ obeys the rules of TOPT in the no-anti-nucleon case [28,31]. The same derivations suffice to prove the result for the scalar field theories defined in the Introduction. It therefore immediately follows that $K_{1}$ is the 2PI TOPT amplitude in the case that only positive-energy states are kept in $G_{0}$. 
We now provide a specific example of the derivation of the time-ordered perturbation theory amplitude by this route. Firstly, it is true in general that, if in Eq. (2.17) $\mathcal{G}$ is replaced by $G_{0}$, then the three-dimensional kernel becomes

$$
K_{1}=\left\langle G_{0}\right\rangle^{-1}\left\langle G_{0} K G_{0}\right\rangle\left\langle G_{0}\right\rangle^{-1} .
$$

We note that this formula was given by Klein in his work on deriving three-dimensional scattering equations from four-dimensional ones [23 25]. Equations (2.17) and (2.9) may thus be thought of as providing a generalization of the formalism of Klein. Secondly, suppose that $K$ is expanded according to the power of the coupling constant in each of its contributions, i.e.,

$$
K=\sum_{i=1}^{\infty} K^{(2 i)}
$$

and for the moment only the second-order, or ladder, contribution to $K$ is kept, i.e., (3.1) is rewritten as:

$$
K_{1}^{(2)}=\left\langle G_{0}\right\rangle^{-1}\left\langle G_{0} K^{(2)} G_{0}\right\rangle\left\langle G_{0}\right\rangle^{-1} .
$$

We now calculate this amplitude in the absence of negative-energy states. First, the propagators are split according to Eq. (1.3), and only the positive-energy pieces retained. Second, the inverse Fourier representations of all quantities are inserted, so that the time-ordering can be clearly elucidated. Third, a change of variables to time differences is made. Fourth, the relative-energy integrations are performed, generating delta functions on some of the time differences. Last, the integrals over these time differences are performed. This procedure allows the calculation of $K_{1}^{(2)}$ in any frame. Indeed, it is completely general, and can be used to calculate $K_{1}$ no matter what $K$ is chosen.

If the total four-momentum of the two-body system in the frame of interest is written $P=(E, \mathbf{P})$, then it is seen that $K_{1}^{(2)}$ takes the standard form of TOPT:

$$
K_{1}^{(2)}\left(\mathbf{p}^{\prime}, \mathbf{p} ; E, \mathbf{P}\right)=\frac{g_{1} g_{2}}{2 \omega} \mathcal{M}\left(\frac{1}{E^{+}-\epsilon_{1}-\epsilon_{2}^{\prime}-\omega}+\frac{1}{E^{+}-\epsilon_{2}-\epsilon_{1}^{\prime}-\omega}\right),
$$

where $E^{+}=E+i \eta, \epsilon_{1}=\epsilon_{1}\left(\mathbf{p}_{1}\right), \epsilon_{2}=\epsilon_{2}\left(\mathbf{p}_{2}\right), \omega=\sqrt{\mu^{2}+\left(\mathbf{p}-\mathbf{p}^{\prime}\right)^{2}}$, and $\epsilon_{1}^{\prime}\left(\epsilon_{2}^{\prime}\right)$ has the argument $\mathbf{p}_{1}^{\prime}\left(\mathbf{p}_{2}^{\prime}\right)$ in place of $\mathbf{p}_{1}\left(\mathbf{p}_{2}\right)$. Here, $m_{1}$ and $m_{2}$ are the the masses of the two interacting particles, $\mu$ is the mass of the exchanged meson and the factor $\mathcal{M}$ is defined by:

$$
\mathcal{M}= \begin{cases}4 m_{1} m_{2}, & \text { for spin-zero particles } \\ 1, & \text { for spin-half particles }\end{cases}
$$

Note that to extract the mass of the bound-state, $M$, one must set $E=\sqrt{M^{2}+\mathbf{P}^{2}}$. The total and relative three-momenta are related to the individual particle momenta in the usual way:

$$
\begin{aligned}
\mathbf{p}_{1}+\mathbf{p}_{2} & =\mathbf{p}_{1}^{\prime}+\mathbf{p}_{2}^{\prime}=\mathbf{P} ; \\
\nu_{2} \mathbf{p}_{1}-\nu_{1} \mathbf{p}_{2} & =\mathbf{p} ; \quad \nu_{2} \mathbf{p}_{1}^{\prime}-\nu_{1} \mathbf{p}_{2}^{\prime}=\mathbf{p}^{\prime} ;
\end{aligned}
$$


where $\nu_{1}$ and $\nu_{2}$ are any two real numbers which obey $\nu_{1}+\nu_{2}=1$. Figure 11 shows the time-ordered diagrams corresponding to Eq. (3.4).

The bound-state equation corresponding to this interaction is then:

$$
\left\langle G_{0}\right\rangle^{-1}\left(\mathbf{p}^{\prime} ; E, \mathbf{P}\right) \psi\left(\mathbf{p}^{\prime} ; E, \mathbf{P}\right)=\int \frac{d^{3} p}{(2 \pi)^{3}} K_{1}^{(2)}\left(\mathbf{p}^{\prime}, \mathbf{p} ; E, \mathbf{P}\right) \psi(\mathbf{p} ; E, \mathbf{P})
$$

where we have omitted the factor of $(2 \pi)^{3} \delta^{(3)}\left(p^{\prime}-p\right)$ from $\left\langle G_{0}\right\rangle$, which integrates out of the equation trivially. Here:

$$
\left\langle G_{0}\right\rangle(\mathbf{p} ; E, \mathbf{P})=\frac{\Lambda_{1}^{+} \Lambda_{2}^{+}}{E+i \eta-\epsilon_{1}-\epsilon_{2}},
$$

where $\Lambda_{1}^{+}=\Lambda_{1}^{+}\left(\mathbf{p}_{1}\right)$ and $\Lambda_{2}^{+}=\Lambda_{2}^{+}\left(\mathbf{p}_{2}\right)$. Now suppose that we have a set of free singleparticle positive-energy momentum-space wave-functions, $u$, for both the spin-half and scalar theories. In the spin-half case these will be the spinors, and their normalization may be chosen such that:

$$
\Lambda^{+}(\mathbf{p})=u(\mathbf{p}) \bar{u}(\mathbf{p})
$$

(where spin indices have been suppressed). In the scalar case Eq. (3.10) may also be enforced, since the $u$ 's may be chosen to be merely numerical factors, i.e., we may define

$$
u(\mathbf{p})=\bar{u}(\mathbf{p}) \equiv \frac{1}{\sqrt{2 \epsilon(\mathbf{p})}} .
$$

Expanding:

$$
\psi(\mathbf{p} ; E, \mathbf{P})=u_{1}\left(\mathbf{p}_{1}\right) u_{2}\left(\mathbf{p}_{2}\right) \phi(\mathbf{p} ; E, \mathbf{P})
$$

(with an implicit summation on spin indices in the spinor case) and manipulating Eq. (3.8) leads to:

$$
\left(E-\epsilon_{1}^{\prime}-\epsilon_{2}^{\prime}\right) \phi\left(\mathbf{p}^{\prime} ; E, \mathbf{P}\right)=\int \frac{d^{3} p}{(2 \pi)^{3}} \tilde{K}_{1}^{(2)}\left(\mathbf{p}^{\prime}, \mathbf{p} ; E, \mathbf{P}\right) \phi(\mathbf{p} ; E, \mathbf{P})
$$

with:

$$
\tilde{K}_{1}^{(2)}\left(\mathbf{p}^{\prime}, \mathbf{p} ; E, \mathbf{P}\right)=\bar{u}_{1}\left(\mathbf{p}_{1}^{\prime}\right) \bar{u}_{2}\left(\mathbf{p}_{2}^{\prime}\right) K_{1}^{(2)}\left(\mathbf{p}^{\prime}, \mathbf{p} ; E, \mathbf{P}\right) u_{1}\left(\mathbf{p}_{1}\right) u_{2}\left(\mathbf{p}_{2}\right) .
$$

Note that the TOPT interaction which appears in the bound-state equation (3.13) changes with total three-momentum of the system. This provides the dynamical boost of the interaction and it is straightforward to show that it incorporates the perturbative boost correction discussed in Ref. [27]. That is, in the equal-mass case, to second order in $\mathbf{P}$ we have (neglecting $\mathbf{p}^{2} / m$ and $\mathbf{p}^{\prime 2} / m$ terms):

$$
\tilde{K}_{1}^{(2)}(\mathbf{q} ; \mathbf{P})=\tilde{K}_{1}^{(2)}(\mathbf{q} ; \mathbf{0})-\frac{\mathbf{P}^{2}}{4 m^{2}} \tilde{K}_{1}^{(2)}(\mathbf{q} ; \mathbf{0})-\frac{1}{8 m^{2}} \mathbf{P} \cdot \mathbf{q} \mathbf{P} \cdot \nabla_{\mathbf{q}} \tilde{K}_{1}^{(2)}(\mathbf{q} ; \mathbf{0}),
$$


where we have written the interaction as a function of the momentum of the exchanged meson, $\mathbf{q}=\mathbf{p}^{\prime}-\mathbf{p}$.

If it is true, as argued in Ref. [27], that this leading-order boost correction is sufficiently accurate for studies of three-nucleon systems, then the boost effects incorporated in the TOPT result (3.4) should be more than adequate. Indeed, we believe such a boost to be preferable to that of Eq. (3.15), since it involves operators which are bounded as $\mathbf{P} \rightarrow \infty$, whereas, at large values of $\mathbf{P}$ the perturbative boost correction (3.15) diverges.

The identification of the kernel $K_{1}$ with the usual time-ordered perturbation theory interaction is only correct in the absence of negative-energy states. With the full fourdimensional propagator, the form of $K_{1}^{(2)}$ obtained from Eq. (3.3) in the manner described above Eq. (3.4) is:

$$
\begin{aligned}
& \left\langle G_{0}\right\rangle\left(\mathbf{p}^{\prime} ; E, \mathbf{P}\right) K_{1}^{(2)}\left(\mathbf{p}^{\prime}, \mathbf{p} ; E, \mathbf{P}\right)\left\langle G_{0}\right\rangle(\mathbf{p} ; E, \mathbf{P})= \\
& \frac{g_{1} g_{2} \mathcal{M}}{2 \omega}\left[\left(\frac{\Lambda_{1}^{+^{\prime}} \Lambda_{2}^{+{ }^{\prime}}}{E-\epsilon_{1}^{\prime}-\epsilon_{2}^{\prime}}+\frac{\Lambda_{1}^{{ }^{\prime}} \Lambda_{2}^{+\prime}}{-\epsilon_{1}^{\prime}-\epsilon_{1}-\omega}\right) \frac{1}{E-\epsilon_{1}-\epsilon_{2}^{\prime}-\omega}\left(\frac{\Lambda_{1}^{+} \Lambda_{2}^{+}}{E-\epsilon_{1}-\epsilon_{2}}+\frac{\Lambda_{1}^{+} \Lambda_{2}^{-}}{-\epsilon_{2}-\epsilon_{2}^{\prime}-\omega}\right)\right. \\
& +\left(\frac{\Lambda_{1}^{+\prime} \Lambda_{2}^{+{ }^{\prime}}}{E-\epsilon_{1}^{\prime}-\epsilon_{2}^{\prime}}+\frac{\Lambda_{1}^{+{ }^{\prime}} \Lambda_{2}^{-\prime}}{-\epsilon_{2}-\epsilon_{2}^{\prime}-\omega}\right) \frac{1}{E-\epsilon_{1}^{\prime}-\epsilon_{2}-\omega}\left(\frac{\Lambda_{1}^{+} \Lambda_{2}^{+}}{E-\epsilon_{1}-\epsilon_{2}}+\frac{\Lambda_{1}^{-} \Lambda_{2}^{+}}{-\epsilon_{1}^{\prime}-\epsilon_{1}-\omega}\right) \\
& +\frac{\Lambda_{1}^{-{ }^{\prime}} \Lambda_{2}^{-\prime}}{-E-\epsilon_{1}^{\prime}-\epsilon_{2}^{\prime}}\left(\frac{1}{-\epsilon_{1}^{\prime}-\epsilon_{1}-\omega}+\frac{1}{-\epsilon_{2}^{\prime}-\epsilon_{2}-\omega}\right) \frac{\Lambda_{1}^{+} \Lambda_{2}^{+}}{E-\epsilon_{1}-\epsilon_{2}} \\
& +\left(\frac{\Lambda_{1}^{-\prime} \Lambda_{2}^{-\prime}}{-E-\epsilon_{1}^{\prime}-\epsilon_{2}^{\prime}}+\frac{\Lambda_{1}^{{ }^{\prime}} \Lambda_{2}^{-\prime}}{-\epsilon_{1}-\epsilon_{1}^{\prime}-\omega}\right) \frac{1}{-E-\epsilon_{1}-\epsilon_{2}^{\prime}-\omega}\left(\frac{\Lambda_{1}^{-} \Lambda_{2}^{-}}{-E-\epsilon_{1}-\epsilon_{2}}+\frac{\Lambda_{1}^{-} \Lambda_{2}^{+}}{-\epsilon_{2}-\epsilon_{2}^{\prime}-\omega}\right) \\
& +\left(\frac{\Lambda_{1}^{-{ }^{\prime}} \Lambda_{2}^{-\prime}}{-E-\epsilon_{1}^{\prime}-\epsilon_{2}^{\prime}}+\frac{\Lambda_{1}^{-\prime} \Lambda_{2}^{+{ }^{\prime}}}{-\epsilon_{2}-\epsilon_{2}^{\prime}-\omega}\right) \frac{1}{-E-\epsilon_{1}^{\prime}-\epsilon_{2}-\omega}\left(\frac{\Lambda_{1}^{-} \Lambda_{2}^{-}}{-E-\epsilon_{1}-\epsilon_{2}}+\frac{\Lambda_{1}^{+} \Lambda_{2}^{-}}{-\epsilon_{1}-\epsilon_{1}^{\prime}-\omega}\right) \\
& \left.+\frac{\Lambda_{1}^{+\prime} \Lambda_{2}^{+^{\prime}}}{E-\epsilon_{1}^{\prime}-\epsilon_{2}^{\prime}}\left(\frac{1}{-\epsilon_{1}^{\prime}-\epsilon_{1}-\omega}+\frac{1}{-\epsilon_{2}^{\prime}-\epsilon_{2}-\omega}\right) \frac{\Lambda_{1}^{-} \Lambda_{2}^{-}}{-E-\epsilon_{1}-\epsilon_{2}}\right] \text {, }
\end{aligned}
$$

where $\Lambda_{i}^{ \pm}=\Lambda_{i}^{ \pm}\left(\mathbf{p}_{i}\right)$ and $\Lambda_{i}^{ \pm \prime}=\Lambda_{i}^{ \pm}\left(\mathbf{p}_{i}^{\prime}\right)$. Note that here, and throughout the rest of this paper, the $i \eta$ prescriptions can be generated by understanding the masses $m_{1}, m_{2}$, and $\mu$ to have a small negative imaginary part.

Now the three-dimensional propagator is

$$
\left\langle G_{0}\right\rangle(\mathbf{p} ; E, \mathbf{P})=\frac{\Lambda_{1}^{+} \Lambda_{2}^{+}}{E+i \eta-\epsilon_{1}-\epsilon_{2}}+\frac{\Lambda_{1}^{-} \Lambda_{2}^{-}}{-E+i \eta-\epsilon_{1}-\epsilon_{2}}
$$

(where once again we have omitted the momentum-space delta-function for notational simplicity), which is the Salpeter propagator [30]. If the negative-energy piece is removed, this becomes the Blankenbecler-Sugar [12] or Logunov-Tavkhelidze [29 propagator. Note that in the spin-half case this propagator does not have a unique inverse, consequently in that case the derivation of the bound-state equation analogous to Eq. (3.13) usually assumes that the +- and -+ pieces of $\left\langle G_{0}\right\rangle^{-1}$ are zero.

The graphs corresponding to the terms in Eq. (3.16) are shown in Figure 2. Graphs (a)(d) are generated by the first line of Eq. (3.16), graphs (e)-(h) by the second line, and graphs (i) and $(\mathrm{j})$ by the third line. The fourth to sixth lines of (3.16) generate the same ten graphs, but with particles and anti-particles interchanged. Note that the graphs drawn in Figure 2 
do not represent actual physical processes, but rather contributions to the "potential" $K_{1}^{(2)}$. As observed by Klein, if negative-energy particles are present, the rules for the construction of this interaction differ from those of standard TOPT [23,24].

In general, it can be shown that the rules for the construction of the full two-body equaltime Green's function, $\left\langle G_{0}+G_{0} T G_{0}\right\rangle$, for the case of positive-energy initial and final-state particles, are, in either of the two field theories given in the Introduction:

1. Draw all topologically distinct, two-to-two graphs, which do not contain vacuumvacuum subdiagrams, remembering that different time-orderings contribute to different graphs.

2. Construct the expression for each individual graph exactly as in TOPT, save that:

(a) All one-particle energies are to be relativistic.

(b) If in some intermediate state of the graph both the initial-state particles are present with exactly the same momenta as in their initial state, i.e., their state is unaltered from the beginning of the graph, then in the TOPT denominator corresponding to that state the quantity $\epsilon_{1}+\epsilon_{2}$ must be replaced by $E$.

(c) Similarly, if both final-state particles are present with exactly the same momenta as in their final state, i.e., they have undergone their last interaction but are still present in some intermediate state, then in the TOPT denominator corresponding to that state the quantity $\epsilon_{1}^{\prime}+\epsilon_{2}^{\prime}$ must be replaced by $E$.

If both these last two conditions are satisfied then the denominator for the relevant state is the TOPT denominator for that state, but with $\epsilon_{1}^{\prime}+\epsilon_{2}^{\prime}+\epsilon_{1}+\epsilon_{2}$ replaced by $2 E$. Note that these are not the rules for the amplitude obtained from the Ladder BSE. The Green's function defined by these rules is the $++\rightarrow++$ piece of the full two-body equal-time Green's function of the field theory.

The substitution of these $E \mathrm{~s}$ in the intermediate-state denominators can be shown to be due to a crucial difference between the way initial and final-states are treated in the calculation of $\left\langle G_{0} T G_{0}\right\rangle$ and in TOPT. In TOPT the initial and final times in the diagram are taken to minus and plus infinity respectively, thus guaranteeing that no interaction takes place before the beginning (or after the end) of the propagation of the two-particle state. However, when $\left\langle G_{0} T G_{0}\right\rangle$ is calculated in our work the initial and final times are kept finite. Consequently the first (last) event in the diagram need not be the creation of the $\phi_{1} \phi_{2}$ or $N_{1} N_{2}$ pair, it may be the creation out of (destruction into) the vacuum of a $\phi \bar{\phi} \sigma$ or $N \bar{N} \sigma$ state. (See for instance the second graph in Figure 2.) The possibility of such an event happening before or after the $\phi_{1} \phi_{2}$ or $N_{1} N_{2}$ propagation in some contributions to $\left\langle G_{0} T G_{0}\right\rangle$ leads to denominators which differ from the TOPT ones in those pieces of $\left\langle G_{0} T G_{0}\right\rangle$. It follows that, if the pieces of the Hamiltonian of the theory which couple $\phi \bar{\phi} \sigma$ or $N \bar{N} \sigma$ states directly to the vacuum are removed from the Hamiltonian, then the modification of the TOPT denominators is not necessary, and the rules for calculation of the two-particle to two-particle part of the Green's function $\left\langle G_{0} T G_{0}\right\rangle$ become exactly those for the calculation of the TOPT Green's function. This equivalence of $\left\langle G_{0} T G_{0}\right\rangle$ and the time-ordered perturbation theory Green's function in the absence of such terms from the 
Hamiltonian was demonstrated for a field theory of nucleons and pions by Kvinikhidze and Blankleider [28].

As discussed in Section [1], the use of a static interaction in the Bethe-Salpeter equation leads to the Salpeter equation (2.14). This equation is often used for the interactions of two relativistic particles. It is straightforward to show that the static limit of the potential given by Eq. (3.16) is the usual instant interaction in the positive-energy sector. On the other hand, Eq. (3.16) predicts that the coupling between the ++ and -- states is suppressed by retardation effects which cause it to be a factor $\omega / m$ (in the equal-mass case) smaller than the rest of the static interaction. This factor is zero in the static limit. Thus, for a causal interaction, the correct static limit of the equation (2.18) with the interaction (3.16) is the Breit equation, i.e., Eq. (2.14) with the -- states omitted from the calculation.

In order to recover the results of Klein for two-meson exchange interactions, and so make connection with the recent work on two-pion exchange of Rijken and Stoks [32, 33] and Lahiff and Afnan [34], we evaluate $\mathcal{G}$ to first order in $K-K_{1}$ in Eq. (2.17). This produces

$$
K_{1}=\left\langle G_{0}\right\rangle^{-1}\left(\left\langle G_{0} K G_{0}\right\rangle+\left\langle G_{0} K G_{0}\left(K-K_{1}\right) G_{0}\right\rangle\right)\left\langle G_{0}\right\rangle^{-1} .
$$

Now expanding $K$ as in Eq. (3.2) and dropping terms of higher than fourth order in the coupling constant leads to

$$
K_{1}^{(4)}=\left\langle G_{0}\right\rangle^{-1}\left(\left\langle G_{0} K^{(4)} G_{0}\right\rangle+\left\langle G_{0} K^{(2)} G_{0} K^{(2)} G_{0}\right\rangle\right)\left\langle G_{0}\right\rangle^{-1}-K_{1}^{(2)}\left\langle G_{0}\right\rangle K_{1}^{(2)} .
$$

When applied to particle-particle scattering Eq. (3.19) gives exactly the same results obtained by Klein, except that where Klein removed the iterated second-order threedimensional interaction by hand, here the formalism provides the subtraction naturally.

The procedure developed in Section [1] thus provides the generalization of Klein's method. The interaction and vertex function defined by Eqs. (2.17), (2.9), and (2.18) have the full relative-energy dynamics of the Bethe-Salpeter equation (2.1) included in them.

\section{A SIMPLE FOUR-DIMENSIONAL EQUATION WITH THE CORRECT ONE-BODY LIMIT}

Now let us return to the four-dimensional BSE (1.1). The simplest BSE with the correct one-body limit is

$$
\Gamma=K_{X} G_{0} \Gamma
$$

where $K_{X}$ is the sum of all 2PI ladder and crossed-ladder two-to-two graphs [11]. The bound-state masses predicted by such an equation have recently been obtained by Monte Carlo integration in the Feynman-Schwinger representation of both scalar $\phi^{3}$ field theory and scalar QED by Nieuwenhuis, Tjon and Simonov 35 39. In general though, the kernel $K_{X}$ is too complicated for (4.1) to be solved by standard means. Therefore, in this section we shall derive a four-dimensional equation which has a simple kernel, the appropriate mesonproduction thresholds and the correct one-body and high-energy (or eikonal) limits. A form of the following derivation appeared in 18 . 
Although it is impossible to rewrite a crossed graph exactly as an iterate of the ladder kernel $K^{(2)}$, the leading contributions of these graphs to the high-energy and one-body limits are iterative. This can be easily shown in the case of the fourth-order crossed-box graph. In both of the field theories defined in the Introduction, this graph corresponds to an expression:

$$
\begin{aligned}
& K_{X}^{(4)}\left(k_{1}^{\prime}, k_{2}^{\prime} ; k_{1}, k_{2}\right)= \\
& \quad i g_{1}^{2} g_{2}^{2} \mathcal{M}^{2} \int \frac{d^{4} p_{2}}{(2 \pi)^{4}} \frac{1}{\left(k_{2}^{\prime}-p_{2}\right)^{2}-\mu^{2}} d_{1}\left(P-p_{2}\right) d_{2}\left(k_{2}+k_{2}^{\prime}-p_{2}\right) \frac{1}{\left(p_{2}-k_{2}\right)^{2}-\mu^{2}} .
\end{aligned}
$$

where the lines have been assigned the momenta shown in Figure 3 , and $P$ is the total momentum, which is conserved:

$$
P=k_{1}+k_{2}=k_{1}^{\prime}+k_{2}^{\prime}
$$

The propagators $d_{i}$ were given in Eq. (1.3), and the factor $\mathcal{M}$ was defined in Eq. (3.5).

Following Wallace and Mandelzweig [18,19] we define, for any four-vector $q$, quantities $q_{\|}$and $q_{\perp \mu}$, as follows:

$$
q_{\mu}=q_{\|} \hat{P}_{\mu}+q_{\perp \mu}
$$

where $\hat{P}$ is the unit four-vector in the direction of $P$ and

$$
q_{\|}=q \cdot \hat{P}
$$

Consequently in Eq. (4.2) the argument of the function $d_{2}$ may be rewritten:

$$
\left(k_{2 \|}+k_{2 \|}^{\prime}-p_{2 \|}\right) \hat{P}+k_{2 \perp}+k_{2 \perp}^{\prime}-p_{2 \perp} .
$$

Now suppose that the momentum of particle 2 is large. This may occur because $m_{2} \gg m_{1}$ (one-body limit) or because particle 2 has very high energy (eikonal limit). In either case its intermediate and final-state momenta will be largely unaffected by the presence of particle 1, and so we may approximate the perpendicular components as unchanging,

$$
k_{2 \perp}+k_{2 \perp}^{\prime} \approx 2 p_{2 \perp} .
$$

Indeed, making the replacement (4.7) in (4.2) will not affect the value of $K_{X}^{(4)}$ in the limit $m_{2} \rightarrow \infty$.

This argument shows that $K_{X}^{(4)}$ may be approximately rewritten as:

$$
\begin{aligned}
& K_{X}^{(4)}\left(k_{2}^{\prime}, k_{2} ; P\right) \approx \\
& \quad i \int \frac{d^{4} p_{2}}{(2 \pi)^{4}} K^{(2)}\left(k_{2}^{\prime}-p_{2}\right) d_{1}\left(P-p_{2}\right) d_{2}\left(\left(k_{2 \|}+k_{2 \|}^{\prime}-p_{2 \|}\right) \hat{P}+p_{2 \perp}\right) K^{(2)}\left(p_{2}-k_{2}\right),
\end{aligned}
$$

where $K^{(2)}$ is the ladder BSE driving term:

$$
K^{(2)}(q)=\mathcal{M} \frac{g_{1} g_{2}}{q^{2}-\mu^{2}+i \eta} .
$$

In operator notation: 


$$
K_{X}^{(4)} \approx K^{(2)} G_{C} K^{(2)}
$$

with

$$
G_{C}\left(p^{\prime}, p ; E_{1}, E_{2}\right)=i(2 \pi)^{4} \delta^{(4)}\left(p^{\prime}-p\right) d_{1}(p) d_{2}\left(\left(E_{2}-E_{1}+p_{\|}\right) \hat{P}-p_{\perp}\right) .
$$

Here $E_{1}$ and $E_{2}$ are defined via:

$$
2 E_{2}=k_{2 \|}+k_{2 \|}^{\prime} ; \quad 2 E_{1}=k_{1 \|}+k_{1 \|}^{\prime} ;
$$

and so in the cm frame $E=E_{1}+E_{2}$. The propagator $G_{C}$ defined by (4.11) therefore depends on the parallel components of the external momenta. Thus the use of operator notation in Eq. (4.10) is not strictly correct. In Eq. (4.27) we redefine $G_{C}$ in order to remove this dependence on external momenta. However, that change in $G_{C}$ modifies the analytic structure of the amplitude defined by the corresponding integral equation. Therefore, for the present we persist with $G_{C}$ defined by (4.11), and use an improper operator notation. Note that if the particles are on-shell in their initial and final states then:

$$
E_{1}=E_{1}^{\mathrm{on}} \equiv \frac{E^{2}+m_{1}^{2}-m_{2}^{2}}{2 E} ; \quad E_{2}=E_{2}^{\mathrm{on}} \equiv \frac{E^{2}+m_{2}^{2}-m_{1}^{2}}{2 E} .
$$

These arguments show that Eq. (4.10) will be exact in the infinite-mass and high-energy limits, thus demonstrating that the pieces of $K_{X}^{(4)}$ which survive in these two limits may, indeed, be written as iterates of $K^{(2)}$.

Now suppose that $K_{X}$ is written as

$$
K_{X}=V+V G_{C} K_{X}
$$

In principle this is always possible, as (4.14) may be taken as a definition of $V$. At second order in the coupling we clearly have:

$$
V^{(2)}=K_{X}^{(2)} \equiv K^{(2)}
$$

while the above argument shows that with this $V^{(2)}$

$$
K_{X}^{(4)}=V^{(2)} G_{C} V^{(2)}
$$

in the high-energy and infinite-mass limits. Thus, a reasonable choice for $V$ is $V=K^{(2)}$. Equation (4.14) then defines corrections to this choice.

Once this $V$ is chosen, Eq. (4.14) and the BSE (4.1) may be combined to yield an "improved" ladder BSE, which, in the two-body cm frame, after a change of variables to total and relative four-momenta, takes the form

$$
\begin{aligned}
\Gamma\left(p_{0}^{\prime}, \mathbf{p}^{\prime} ; s\right)= & i \int \frac{d^{4} p}{(2 \pi)^{4}} K^{(2)}\left(p^{\prime}-p\right) d_{1}\left(E_{1}^{\mathrm{On}}+p_{0}, \mathbf{p}\right) \\
& {\left[d_{2}\left(E_{2}^{\mathrm{On}}-p_{0},-\mathbf{p}\right)+d_{2}\left(E_{2}^{\mathrm{On}}-p_{0}^{\prime}+p_{0},-\mathbf{p}\right)\right] \Gamma\left(p_{0}, \mathbf{p} ; s\right) . }
\end{aligned}
$$

We stress that what has been done here is to take certain pieces of the Bethe-Salpeter kernel $K_{X}$ and rewrite them in the form $K^{(2)} G_{C} K^{(2)}, K^{(2)} G_{C} K^{(2)} G_{C} K^{(2)}$, etc. Consequently, 
Eq. (4.17) is equivalent to a Bethe-Salpeter equation in which graphs other than one-meson exchange are approximately included in the kernel. Thus we expect that the solution of this equation may provide a better description of the dynamics of two-particle systems than the ladder BSE amplitude.

This will be especially true for systems with one particle much heavier than the other. In these systems the one-body limit constitutes an important piece of the dynamics. Unlike the ladder BSE, Eq. (4.17) has the correct one-body limit. This may be shown as follows. For the on-shell vertex function $p_{0}^{\prime}=0$. If $p_{0}^{\prime}=0$ the sum of the two particle 2 propagators, multiplied by $\mathcal{M}$ is, in the spin-zero case:

$\mathcal{M}\left[d_{2}\left(E_{2}^{\mathrm{on}}-p_{0},-\mathbf{p}\right)+d_{2}\left(E_{2}^{\mathrm{on}}+p_{0},-\mathbf{p}\right)\right]=\frac{4 m_{1} m_{2}}{\left(E_{2}^{\mathrm{On}}-p_{0}\right)^{2}-\epsilon_{2}^{2}+i \eta}+\frac{4 m_{1} m_{2}}{\left(E_{2}^{\mathrm{On}}+p_{0}\right)^{2}-\epsilon_{2}^{2}+i \eta}$,

while for the spin-half case the same combination becomes:

$$
\frac{\left(E_{2}^{\mathrm{on}}-p_{0}\right) \gamma^{0}+\gamma_{2} \cdot \mathbf{p}+m_{2}}{\left(E_{2}^{\mathrm{On}}-p_{0}\right)^{2}-\epsilon_{2}^{2}+i \eta}+\frac{\left(E_{2}^{\mathrm{on}}+p_{0}\right) \gamma^{0}+\gamma_{2} \cdot \mathbf{p}+m_{2}}{\left(E_{2}^{\mathrm{On}}+p_{0}\right)^{2}-\epsilon_{2}^{2}+i \eta} .
$$

In the $m_{2} \rightarrow \infty$ limit, $E_{2}^{\mathrm{On}} \rightarrow \epsilon_{2} \rightarrow m_{2}$, and so this expression reduces to

$$
\left[\frac{1}{p_{0}+i \eta}-\frac{1}{p_{0}-i \eta}\right] 2 m_{1}=-2 \pi i \delta\left(p^{0}\right) 2 m_{1},
$$

for the spin-zero case and

$$
-2 \pi i \delta\left(p^{0}\right) \Lambda_{2 \infty}^{+}
$$

for the spin-half case, where

$$
\Lambda_{2 \infty}^{+}=\frac{1+\gamma_{0}}{2}
$$

is the positive-energy projection operator for an infinitely massive particle two. Thus, in the infinite $m_{2}$ limit Eq. (4.17) yields the Klein-Gordon or Dirac equation for the wave function of particle 1 moving in the static $\sigma$ field generated by particle 2

$$
\frac{E^{2}-m_{1}^{2}-\mathbf{p}^{\prime 2}}{2 m_{1}} \psi\left(\mathbf{p}^{\prime}\right)=-\int \frac{d^{3} p}{(2 \pi)^{3}} \frac{g_{1} g_{2}}{\left(\mathbf{p}^{\prime}-\mathbf{p}\right)^{2}-\mu^{2}} \psi(\mathbf{p}),
$$

or

$$
\left(E \gamma^{0}-\mathbf{p}^{\prime} \cdot \gamma-m_{1}\right) \psi\left(\mathbf{p}^{\prime}\right)=-\int \frac{d^{3} p}{(2 \pi)^{3}} \frac{g_{1} g_{2}}{\left(\mathbf{p}^{\prime}-\mathbf{p}\right)^{2}-\mu^{2}} \psi(\mathbf{p})
$$

where

$$
\psi(\mathbf{p})=\frac{1}{E^{2}-m_{1}^{2}-\mathbf{p}^{2}} \Gamma(0, \mathbf{p} ; E)
$$

or 


$$
\psi(\mathbf{p})=\frac{1}{E \gamma^{0}-\mathbf{p} \cdot \gamma-m_{1}} \Lambda_{2 \infty}^{+} \Gamma(0, \mathbf{p} ; E)
$$

and $E=E_{1}^{\mathrm{on}}$.

Equation (4.17) also has the appropriate meson-production thresholds, and therefore could be used as a basis for four-dimensional calculations of two-body bound-state properties. However, the equation cannot be written in the form

$$
\Gamma=K^{(2)} G \Gamma
$$

and consequently the method of Section III cannot be applied to it. Therefore, we now seek an approximate version of Eq. (4.17) which can be written in the form (4.25).

In the on-shell vertex function $p_{0}^{\prime}=0$. In what follows we use $p_{0}^{\prime}=0$ in the integrand also when the amplitude is not on-shell. This approximation provides a four-dimensional version of the three-dimensional Wallace-Mandelzweig equation obtained in Ref. [17 [19]. In the center-of-mass frame it is

$$
\begin{aligned}
\Gamma\left(p^{\prime} ; E_{1}^{\mathrm{on}}, E_{2}^{\mathrm{on}}\right)= & i \int \frac{d^{4} p}{(2 \pi)^{4}} K^{(2)}\left(p^{\prime}-p\right) d_{1}\left(E_{1}^{\mathrm{on}}+p_{0}, \mathbf{p}\right) \\
& {\left[d_{2}\left(E_{2}^{\mathrm{on}}-p_{0},-\mathbf{p}\right)+d_{2}\left(E_{2}^{\mathrm{on}}+p_{0},-\mathbf{p}\right)\right] \Gamma\left(p ; E_{1}^{\mathrm{On}}, E_{2}^{\mathrm{on}}\right) . }
\end{aligned}
$$

Redefining $G_{C}$ to be, in the cm frame,

$$
G_{C}\left(p^{\prime}, p ; E_{1}^{\mathrm{On}}, E_{2}^{\mathrm{On}}\right)=i(2 \pi)^{4} \delta^{(4)}\left(p^{\prime}-p\right) d_{1}\left(E_{1}^{\mathrm{On}}+p_{0}, \mathbf{p}\right) d_{2}\left(E_{2}^{\mathrm{On}}+p_{0},-\mathbf{p}\right),
$$

allows Eq. (4.26) to be written:

$$
\Gamma=K^{(2)}\left(G_{0}+G_{C}\right) \Gamma .
$$

The following points are worth noting:

1. Because the new choice for $G_{C}$ does not affect the on-shell fourth-order piece of the amplitude Eq. (4.10) is still exactly true on-shell in the one-body and eikonal limits.

2. In the limit $m_{2} \rightarrow \infty$ Eq. (4.26) has the correct one-body limit, as can be seen by a similar argument to that given for Eq. (4.17).

3. If a pinch analysis of the singularities of the amplitude $T$ defined by the scattering equation corresponding to (4.26) is performed then it is found that at order $g^{4}$ there is a production cut in the $p_{0}^{\prime}$ plane of the half-off-shell amplitude which extends upwards from

$$
p_{0}^{\prime}=m_{2}+\mu-E_{2}^{\mathrm{on}},
$$

and lies infinitessimally below the real axis. In the on-shell amplitude at sixth order this cut appears in the integrand and overlaps with the pole of the Green's function at $p_{0}^{\prime}=E_{2}^{\mathrm{On}}-\epsilon_{2}(\mathbf{q})$, so producing a cut in the $E_{2}^{\mathrm{On}}$ plane of $T$ which extends upwards from 


$$
E_{2}^{\mathrm{on}}=m_{2}+\frac{\mu}{2}
$$

This is not a particle-production threshold which exists in the full Bethe-Salpeter scattering amplitude. Hence its existence in the amplitude $T$ defined by the scattering equation corresponding to Eq. (4.26) must be regarded as a deficiency of that equation. It arises because the method used to derive Eq. (4.26) is only guaranteed to produce the correct cut-structure for the second and fourth-order on-shell amplitudes. It should be noted that the vertex function given by Eq. (4.17) does not contain this anomalous threshold.

The value of $E$ at which the threshold (4.30) occurs in the on-shell amplitude is

$E=m_{2}+\frac{\mu}{2}+\sqrt{m_{1}^{2}+m_{2} \mu+\frac{\mu^{2}}{4}}$. In fact, since Equation (4.17) was designed for use in the $m_{2}>m_{1}$ regime this is actually above $E=m_{1}+m_{2}+\mu$. Therefore the theory has the correct threshold structure

for all energies $E$ such that $m_{2}-m_{1}<E<m_{1}+m_{2}+\mu$. Furthermore, if $m_{1}=m_{2}$ then the threshold (4.30) actually lies at $E=m_{1}+m_{2}+\mu$, which is where the first production threshold of the crossed-box graph should be. Consequently, we think of this cut as representing the usual single-pion-production threshold of the crossed-box graph, but somewhat displaced if $m_{2}>m_{1}$.

4. This equation was derived for particles of different mass. If we desire an equation for identical particles a symmetric form of the propagator $G_{C}$ must be used. (We shall return to this point in the next section.)

5. The above derivation could equally well be pursued for the exchange of vector particles. However, in, for instance, scalar QED, account must be taken of the seagull graphs. Furthermore, in the case of spinor QED more care must be taken, since an additional piece of the interaction may be generated when one attempts to write $X \approx V G_{C} V$. (The interested reader may consult [19] for details on these points.)

\section{A THREE-DIMENSIONAL EQUATION WITH RETARDATIONS AND THE CORRECT ONE-BODY LIMIT}

We now apply the relative-energy integration method of Section [1] to the fourdimensional equation (4.26). By so doing we first recover Wallace and Mandelzweig's original quasi-potential equation, and then calculate a three-dimensional kernel which includes firstorder retardation corrections to this result.

Observe that once $G_{C}$ is defined by Eq. (4.27), Eqs. (4.14) and (4.1) are equivalent to:

$$
\Gamma=V\left(G_{0}+G_{C}\right) \Gamma
$$

where $V$ is regarded as being defined by (4.14). This provides a method for calculating corrections to Eq. (4.28), which was obtained by assuming that $V=K^{(2)}$. The result of applying the method of Section 1 to Eq. (5.1) is:

$$
\begin{aligned}
\Gamma_{1} & =V_{1}\langle\mathcal{G}\rangle \Gamma_{1}, \\
\mathcal{G} & =G_{0}+G_{C}+\left(G_{0}+G_{C}\right)\left(V-V_{1}\right) \mathcal{G} .
\end{aligned}
$$


If $V$ is taken to be $V_{\sigma \text { inst }}$, where $V_{\sigma \text { inst }}$ is the static one-sigma exchange potential,

$$
V_{\sigma \text { inst }}\left(\mathbf{p}^{\prime}, \mathbf{p}\right)=-\frac{g_{1} g_{2} \mathcal{M}}{\left(\mathbf{p}-\mathbf{p}^{\prime}\right)^{2}+\mu^{2}}
$$

these equations reduce to

$$
\Gamma_{1}=V_{\sigma \mathrm{inst}} G_{E T} \Gamma_{1}
$$

which is a three-dimensional equation with a free two-body propagator that in the cm frame takes the form:

$$
\begin{aligned}
G_{E T}\left(\mathbf{p} ; E_{1}^{\mathrm{on}}, E_{2}^{\mathrm{on}}\right) \equiv & \left\langle G_{0}+G_{C}\right\rangle \\
= & \frac{\Lambda_{1}^{+} \Lambda_{2}^{+}}{E_{1}^{\mathrm{On}}+E_{2}^{\mathrm{On}}-\epsilon_{1}-\epsilon_{2}+i \eta}+\frac{\Lambda_{1}^{+} \Lambda_{2}^{-}}{E_{1}^{\mathrm{On}}-E_{2}^{\mathrm{On}}-\epsilon_{1}-\epsilon_{2}+i \eta} \\
& \quad+\frac{\Lambda_{1}^{-} \Lambda_{2}^{+}}{-E_{1}^{\mathrm{On}}+E_{2}^{\mathrm{On}}-\epsilon_{1}-\epsilon_{2}+i \eta}+\frac{\Lambda_{2}^{-}}{-E_{1}^{\mathrm{On}}-E_{2}^{\mathrm{On}}-\epsilon_{1}-\epsilon_{2}+i \eta}
\end{aligned}
$$

where once again a factor of $(2 \pi)^{3} \delta^{(3)}\left(p^{\prime}-p\right)$ has been omitted for notational simplicity. Note that this propagator is equivalent to that derived by Cooper and Jennings by a different technique 40]. Note also that in the case $m_{1}=m_{2}$ this same propagator is referred to as the equal-time propagator by Tjon and collaborators [15, 37, 38, 41, 42, .

The desired generalization of Eq. (5.5) which incorporates retardation and boost effects follows from applying the ideas of Sections II and III to Eq. (5.3). If $V_{1}$ is chosen such that the last term in Eq. (5.3) is zero, the formula

$$
V_{1}=G_{E T}^{-1}\left\langle\left(G_{0}+G_{C}\right) V \mathcal{G}\right\rangle G_{E T}^{-1}
$$

is obtained for the general three-dimensional interaction to be used in

$$
\Gamma_{1}=V_{1} G_{E T} \Gamma_{1}
$$

A first step in the inclusion of retardation and boost effects may be taken by following Klein's work and replacing $\mathcal{G}$ by $G_{0}+G_{C}$. This yields what we refer to as the first-order relative-energy integration result:

$$
\begin{aligned}
V_{1} & =G_{E T}^{-1} A G_{E T}^{-1} \\
A & =\left\langle\left(G_{0}+G_{C}\right) V\left(G_{0}+G_{C}\right)\right\rangle .
\end{aligned}
$$

We now take $V$ equal to $K^{(2)}$ and calculate $A$ in the center-of-mass frame. In order to do this we first decompose the cm frame propagators:

$$
d_{i}(p)=\sum_{\rho_{i}} \frac{\Lambda_{i}^{\rho_{i}}(\mathbf{p})}{\rho_{i} p_{0}-\epsilon_{i}(\mathbf{p})} ; \quad i=1,2,
$$

where $\rho_{i}$ may take on the values \pm 1 . It follows that the amplitude $A$ may be written as

$$
A=\sum_{\rho_{1} \rho_{1}^{\prime} \rho_{2} \rho_{2}^{\prime}} \Lambda_{1}^{\rho_{1}^{\prime}{ }^{\prime}} \Lambda_{2}^{\rho_{2}^{\prime} \prime} A\left(\rho_{1}^{\prime} \rho_{2}^{\prime} \leftarrow \rho_{1} \rho_{2}\right) \Lambda_{1}^{\rho_{1}} \Lambda_{2}^{\rho_{2}} .
$$


The sixteen contributions $A\left(\rho_{1}^{\prime} \rho_{2}^{\prime} \leftarrow \rho_{1} \rho_{2}\right)$ may be calculated by the same method used to obtain the Klein potential (3.16). They are found to take on a slightly different form depending on whether $\rho_{1}^{\prime} \rho_{1}$ is equal to plus or minus one. In order to simplify the expressions for the $A$ 's we define the following quantities:

$$
\begin{gathered}
F=\frac{g_{1} g_{2} \mathcal{M}}{2 \omega} ; \\
e_{i}=\rho_{i} E_{i}^{\mathrm{On}}-\epsilon_{i} ; \quad e_{i}^{\prime}=\rho_{i}^{\prime} E_{i}^{\mathrm{On}}-\epsilon_{i}^{\prime} ; \quad i=1,2 .
\end{gathered}
$$

If $\rho_{1}^{\prime} \rho_{1}=1$ then:

$$
\begin{aligned}
A\left(\rho_{1}^{\prime} \rho_{2}^{\prime} \leftarrow \rho_{1} \rho_{2}\right)\left(\mathbf{p}^{\prime}, \mathbf{p} ; E_{1}^{\mathrm{on}}, E_{2}^{\mathrm{on}}\right) & =F \frac{1}{e_{1}^{\prime}+e_{2}^{\prime}} \frac{1}{e_{1}+e_{2}^{\prime}-\omega}\left[\frac{1}{e_{1}+e_{2}}+\frac{1}{e_{2}+e_{2}^{\prime}-\omega}\right] \\
& +F\left[\frac{1}{e_{1}^{\prime}+e_{2}^{\prime}}+\frac{1}{e_{2}+e_{2}^{\prime}-\omega}\right] \frac{1}{e_{1}^{\prime}+e_{2}-\omega} \frac{1}{e_{1}+e_{2}}
\end{aligned}
$$

Conversely, if $\rho_{1}^{\prime} \rho_{1}=-1$ then:

$$
\begin{aligned}
A\left(\rho_{1}^{\prime} \rho_{2}^{\prime} \leftarrow \rho_{1} \rho_{2}\right)\left(\mathbf{p}^{\prime}, \mathbf{p} ; E_{1}^{\mathrm{on}}, E_{2}^{\mathrm{on}}\right) & =F \frac{1}{e_{1}+e_{1}^{\prime}-\omega} \frac{1}{e_{1}+e_{2}^{\prime}-\omega}\left[\frac{1}{e_{1}+e_{2}}+\frac{1}{e_{2}+e_{2}^{\prime}-\omega}\right] \\
& +F\left[\frac{1}{e_{1}^{\prime}+e_{2}^{\prime}}+\frac{1}{e_{2}+e_{2}^{\prime}-\omega}\right] \frac{1}{e_{1}^{\prime}+e_{2}-\omega} \frac{1}{e_{1}+e_{1}^{\prime}-\omega} \\
& +F \frac{1}{e_{1}^{\prime}+e_{2}^{\prime}}\left[\frac{1}{e_{1}+e_{1}^{\prime}-\omega}+\frac{1}{e_{2}+e_{2}^{\prime}-\omega}\right] \frac{1}{e_{1}+e_{2}} .
\end{aligned}
$$

We note the following points about the interaction defined by Eqs. (5.7), (5.10), (5.13), (5.16) and (5.17).

1. As expected from the four-dimensional equation (4.26) it assumes a static form in the limit $m_{2} \rightarrow \infty$. In other words, it has the correct one-body limit. (Recall that we assumed that particle 2 was the heavier of the two.)

2. In the static limit of the interaction defined by Eqs. (5.16) and (5.17) all couplings to the -- states are suppressed. On the other hand, the other couplings tend to the same static limit as the $++\rightarrow++$ piece of the interaction. Thus, in this limit the $++\rightarrow+-$ coupling, which is due to Z-graphs, is correctly given by the instantaneous exchange interaction, and only the -- states need be omitted from a calculation. The role of the +- and -- states in this limit is therefore consistent with the one-body limit which motivated Eq. (5.9).

3. Also as expected from (4.26) $A(++\leftarrow++)$ has a singularity structure which is different to that of the ordinary time-ordered perturbation theory amplitude: it contains a cut beginning at $E_{2}=m_{2}+\frac{\mu}{2}$. The same comments made about the unusual singularity structure of (4.26) apply to this cut.

4. Because we have used a form of $G_{C}$ derived by considering the limit $m_{2} \rightarrow \infty$, the interaction is not symmetric under the interchange of particle 1 and 2 labels. 
Points (2) and (3) are not a reflection of the underlying physics of the meson-exchange process, but rather of the particular iterative form we used in our attempt to sum some of the higher-order graphs in the kernel of the BSE (4.1).

The choice of $G_{C}$ used above involves an approximation which is appropriate in the regime $m_{2}>m_{1}$. For equally massive particles a more appropriate choice for the propagator $G_{C}$ is:

$$
\begin{aligned}
G_{C}\left(p^{\prime}, p ; E_{1}^{\mathrm{on}}, E_{2}^{\mathrm{on}}\right)=i(2 \pi)^{4} \delta^{(4)}\left(p^{\prime}-p\right) & \frac{1}{2}\left[d_{1}\left(E_{1}^{\mathrm{on}}+p_{0}, \mathbf{p}\right) d_{2}\left(E_{2}^{\mathrm{on}}+p_{0},-\mathbf{p}\right)\right. \\
& \left.+d_{1}\left(E_{1}^{\mathrm{on}}-p_{0}, \mathbf{p}\right) d_{2}\left(E_{2}^{\mathrm{on}}-p_{0},-\mathbf{p}\right)\right] .
\end{aligned}
$$

Note that this choice is label symmetric, and hence the resulting bound-state equation may be used for identical particles. The $G_{C}$ defined by (4.27) did not have this property.

When Eqs. (5.10) and (5.11) are applied with this symmetrized form of $G_{C}$ the result is a $V_{1}$ of the form (5.13), but with an $A\left(\rho_{1}^{\prime} \rho_{2}^{\prime} \leftarrow \rho_{1} \rho_{2}\right)$ which may be written in any $\rho$-spin channel as:

$$
\begin{aligned}
& A\left(\mathbf{p}^{\prime}, \mathbf{p} ; E_{1}^{\mathrm{on}}, E_{2}^{\mathrm{on}}\right)= \\
& \frac{F}{2}\left[\frac{1}{e_{1}^{\prime}+e_{2}^{\prime}}\left(\frac{1}{e_{1}+e_{2}^{\prime}-\omega}+\frac{1}{e_{1}^{\prime}+e_{2}-\omega}+\frac{1}{e_{1}+e_{1}^{\prime}-\omega}+\frac{1}{e_{2}+e_{2}^{\prime}-\omega}\right) \frac{1}{e_{1}+e_{2}}\right. \\
& \quad+\frac{1}{e_{1}^{\prime}+e_{2}^{\prime}} \frac{1}{e_{1}+e_{2}^{\prime}-\omega} \frac{1}{e_{2}+e_{2}^{\prime}-\omega}+\frac{1}{e_{1}^{\prime}+e_{2}^{\prime}} \frac{1}{e_{1}^{\prime}+e_{2}-\omega} \frac{1}{e_{1}+e_{1}^{\prime}-\omega} \\
& \quad+\frac{1}{e_{1}+e_{1}^{\prime}-\omega} \frac{1}{e_{1}+e_{2}^{\prime}-\omega} \frac{1}{e_{1}+e_{2}}+\frac{1}{e_{2}+e_{2}^{\prime}-\omega} \frac{1}{e_{1}^{\prime}+e_{2}-\omega} \frac{1}{e_{1}+e_{2}} \\
& \left.\quad+\frac{1}{e_{2}+e_{2}^{\prime}-\omega}\left(\frac{1}{e_{1}+e_{2}^{\prime}-\omega}+\frac{1}{e_{1}^{\prime}+e_{2}-\omega}\right) \frac{1}{e_{1}+e_{1}^{\prime}-\omega}\right],
\end{aligned}
$$

with all symbols defined as above.

\section{COMPARISON OF THE BOUND-STATE PREDICTIONS OF DIFFERENT THREE AND FOUR-DIMENSIONAL INTEGRAL EQUATIONS}

Having derived the interaction which is to be used in Eq. (5.9) we may now compare the two-body bound-state properties predicted by this integral equation with those properties obtained from other three and four-dimensional calculations. This is done in the scalar field theory defined in the Introduction. The masses $m_{1}, m_{2}$, and $\mu$ are chosen to be $m_{1}=m_{2}=m$ and $\mu=0.15 \mathrm{~m}$. Units are then chosen so that $\hbar=c=m=1$.

The three-dimensional equations to be considered are:

1. The Salpeter equation, which may be written:

$$
\Gamma_{S}=V_{\sigma \text { inst }}\left\langle G_{0}\right\rangle \Gamma_{S},
$$

with $V_{\sigma \text { inst }}$ given by Eq. (5.4) and $\left\langle G_{0}\right\rangle$ by Eq. (3.17). Note that in this scalar field theory Eq. (6.1) is exactly equivalent to the Blankenbecler-Sugar equation obtained from the ladder BSE. 
2. The Klein equation for a one-sigma-exchange interaction, which is Eq. (2.18) with the interaction defined by Eqs. (3.16) and (3.17).

3. The equation proposed by Wallace and Mandelzweig, which we refer to as the ET equation:

$$
\Gamma_{E T}=V_{\sigma \mathrm{inst}} G_{E T} \Gamma_{E T}
$$

where $G_{E T}$ was given in Eq. (5.7).

4. The retarded ET equation, Eq. (5.2), with the label-symmetric interaction obtained in the previous section, i.e., with $V_{1}$ defined by (5.19), (5.13), (5.10) and (5.7)

The four-dimensional equations these four calculations are to be compared to are:

1. The ladder Bethe-Salpeter equation.

2. Equation (4.26) which, unlike the ladder BSE, has the correct one-body limit.

We consider the six bound-state equations as falling into two groups: the first contains the ladder BSE and the two three-dimensional reductions thereof: the Salpeter equation and the Klein equation. The second group contains the four-dimensional Wallace-Mandelzweig equation (4.26) and the two three-dimensional equations derived from it: the equal-time equation (5.5) and the first-order relative-energy integration equation (5.9) with the interaction $V_{1}$ defined by Eqs. (5.19), (5.13), (5.10) and (5.7). Within these two groups the three equations may then be thought of as being zeroth (instant potential equations), first (Kleintype) and infinite (four-dimensional equations) order relative-energy integration results.

At any given bound-state mass the equation:

$$
\Gamma(m)=M(m) \Gamma(m),
$$

whether it be a three or four-dimensional integral equation, is an eigenvalue problem. The coupling constant $g_{1} g_{2}$ which appears in the kernel must be chosen such that $M(m)$ has eigenvalue one. If we are searching for the ground-state of the system then we may assume that $\Gamma$ is an $S$-wave state, thus implying that the function $\Gamma$ has no angular dependence. The integration over $\hat{p}$ may then be easily performed. The resultant one (or two) dimensional integral equation may then be discretized, reducing the problem to one of solving for the eigenvalues of a matrix version of $M$. Note that in the four-dimensional equations it is necessary to perform a Wick rotation in the variable $p_{0}$ before the kernel $M$ is discretized, as otherwise the analytic structure in the kernel makes this procedure numerically unstable.

When this approach is implemented for the equations listed above the six bound-state spectra shown in Figure 1 are obtained. The following observations may immediately be made:

1. The two instant formalisms (Salpeter and ET) predict more binding at a given coupling than either their corresponding four-dimensional equation or their corresponding Klein-type interaction. 
2. The calculations in the second group always predict deeper binding than the corresponding calculations in the first group. In other words, the inclusion of pieces of higher-order graphs in the kernel of the integral equation yields deeper binding at a given coupling.

3. The first-order relative-energy integration (Klein-type) interactions give predictions for the bound-state spectra which are significantly closer to those of the full fourdimensional calculation than the spectra of the instant equations.

All of these results can be understood on physical grounds. Point 1 arises because the inclusion of retardation in the scalar field theory always reduces the amount of attraction in the bound-state. An example of this is provided by one-sigma exchange. When the full retardation corrections predicted by time-ordered perturbation theory are included the instant potential (5.4) is replaced by the retarded interaction (3.4) (provided the effects of negative-energy states are ignored). If $E<m_{1}+m_{2}$ this will always be a negative number that is smaller in magnitude than the instant potential. Meanwhile, the inclusion of pieces of additional higher-order graphs leads to deeper binding since in the $\phi^{2} \sigma$ theory all timeordered perturbation theory graphs are attractive in the bound-state region. Most significant though, in our opinion, is point 3, which shows that the first-order relative-energy integration (Klein-type) potentials do a better job than the instant interactions of approximating the four-dimensional equation which both are derived from.

All six of these calculations aim to sum the main contributions to the two-to-two amplitude. However, Figure 4 shows that they give different results, and so they cannot all be correct. The question therefore arises: what is the binding energy at a given coupling if the full scattering series is summed? A partial answer to this question was recently provided by Nieuwenhuis and Tjon, who summed the series of all ladder and crossed-ladder diagrams using Monte Carlo integration techniques in the Feynman-Schwinger representation, and so obtained two-body bound-state masses in this scalar theory [35 38]. Their results, calculated at six different coupling strengths, show that the sum of all such graphs predicts deeper binding than any of our six integral equation calculations. In particular, the ET appears to do the best job of reproducing the bound-state spectrum obtained by Nieuwenhuis and Tjon. At first sight this appears to recommend the ET as the best formalism for doing three-dimensional calculations. However, two points must be remembered before a definite conclusion is drawn.

Firstly, the reason that the Nieuwenhuis-Tjon calculation predicts so much more binding than the two four-dimensional integral equation calculations performed here is precisely the fact discussed in connection with point 2 above: in the $\phi^{2} \sigma$ theory all time-ordered perturbation theory graphs give attraction in the bound-state region. Hence the NieuwenhuisTjon calculation, which includes many more such graphs than both the ladder BSE and four-dimensional Wallace-Mandelzweig equation calculations, must predict more binding. Therefore, we claim that the Salpeter and ET equations do a "better" job of imitating the full Nieuwenhuis-Tjon calculation than the ladder BSE and four-dimensional WallaceMandelzweig equation because instant calculations such as the Salpeter and ET ones ignore two competing effects.

1. Higher-order graphs are left out of their kernel. If included these graphs would lead to deeper binding at a given coupling. 
2. Exchanged-meson retardation is completely ignored. If included this retardation would lead to shallower binding at a given coupling.

In other words, we claim that the ET formalism's apparent success is due to the cancellation of these two neglected effects and cannot necessarily be interpreted as a signal of that approach having the "right physics" for the problem.

Secondly, none of these calculations have been performed in the way that calculations in few-body hadronic physics are usually performed. When such bound-state calculations are done in hadronic physics one usually acknowledges that the use of any integral equation necessarily means the neglect of some graphs of the theory, and so one regards the couplings that appear in that equation as effective. These couplings are then usually fixed by fitting to some set of observables, and then certain different observables are predicted. This makes the connection to an underlying field theory somewhat more tenuous, but it does allow calculation to proceed in situations where:

- The value of the coupling which appears in the "fundamental" Lagrangian is not known.

- The problem is highly non-perturbative and consequently any truncation is somewhat suspect.

Therefore, to test the procedure of Section $\amalg$ for deriving three-dimensional integral equations in a way consistent with that in which it would be used in hadronic physics, we fix the mass of the bound-state at $M=1.95$ and calculate the wave-functions

$$
\psi\left(\mathbf{p}^{\prime} ; E\right)=g\left(\mathbf{p}^{\prime} ; E\right) \Gamma\left(\mathbf{p}^{\prime} ; E\right),
$$

for each of the four different three-dimensional calculations. (Here $g$ is the free two-particle Green's function for the relevant calculation.) Each three-dimensional wave-function must be normalized according to

$$
\int \frac{d^{3} p^{\prime} d^{3} p}{(2 \pi)^{6}} \psi^{*}\left(\mathbf{p}^{\prime} ; E\right) \frac{\partial G^{-1}}{\partial E}\left(\mathbf{p}^{\prime}, \mathbf{p} ; E\right) \psi(\mathbf{p} ; E)=2 E,
$$

where $G$ is the full two-particle Green's function appearing in the three-dimensional equation from which $\Gamma$ was obtained. These wave-functions are then compared to the four-dimensional wave functions, integrated over the zeroth component of the relative four-momentum:

$$
\psi\left(\mathbf{p}^{\prime} ; E\right)=\frac{1}{2 \pi} \int d p_{0}^{\prime} g\left(p_{\mu}^{\prime} ; E\right) \Gamma\left(p_{\mu}^{\prime} ; E\right) .
$$

Here $g$ is the appropriate free two-particle four-dimensional Green's function. The fourdimensional vertex function used in (6.6) is normalized according to:

$$
\int \frac{d^{4} p^{\prime} d^{4} p}{(2 \pi)^{8}} \Gamma^{*}\left(p_{\mu}^{\prime} ; E\right) g\left(p_{\mu}^{\prime} ; E\right) \frac{\partial G^{-1}}{\partial E}\left(p_{\mu}^{\prime}, p_{\mu} ; E\right) g\left(p_{\mu} ; E\right) \Gamma\left(p_{\mu} ; E\right)=2 E,
$$

where $G$ is the full two-particle Green's function appearing in the four-dimensional equation from which $\Gamma$ was obtained. The left-hand sides of Eqs. (6.5) and (6.7) may be reduced 
to two (and four)-dimensional integrals using the fact that the $S$-wave wave-function $\psi$ is angle-independent. Note that in the four-dimensional case, Wick rotation in the zeroth component allows use of the vertex function on the $p_{0}$ or $p_{0}^{\prime}$ imaginary axis.

When the normalized wave-functions of the equations in group 1 (group 2) are compared the results shown in Figure 5 (6) are obtained. It is immediately seen that all the wave-functions at this bound-state mass have essentially the same features. However, in both figures it is clear that the three-dimensional equation with the first-order relativeenergy integration, or Klein-type, interaction reproduces the integrated four-dimensional wave function considerably more accurately than the instant calculation does. This shows that the procedure given in Section 2 does provide a way to systematically derive threedimensional equations which are closer to their four-dimensional counterparts than those which are obtained from merely adopting an instant interaction.

\section{CONCLUSION}

Quasi-potential equations (QPEs), despite their success as a basis for hadronic phenomenology, cannot be improved upon systematically. This is because whenever they are used beyond first-order they predict amplitudes with unphysical singularities. These singularities also appear whenever attempts are made to boost the interaction appearing in the QPE from one frame to another. They arise from these equations' use of delta-function constraints on the relative four-momentum.

Because of this shortcoming of QPEs, in Section [1] of this paper we sought, and found, a systematic procedure for deriving three-dimensional equations from four-dimensional ones which does not involve the use of delta functions. As shown in Section III, this procedure is akin to the work of Klein. It allows the derivation of a three-dimensional kernel which includes, in a systematic expansion which may be pursued to any desired accuracy, the effects of the relative-energy integration present in the four-dimensional integral equation. If the procedure is applied to infinite order, a result equivalent to the original four-dimensional equation is obtained. At any order the kernel derived has a simple interpretation in terms of the diagrams of time-ordered perturbation theory, except that the presence of negativeenergy states requires additional rules. The resultant three-dimensional equations therefore include more of the meson-retardation effects than quasi-potential equations, and incorporate the effects of a dynamical boost.

Since the BSE with any kernel which is a finite sum of Feynman graphs does not have the correct one-body limit, if the procedure of Section प1] is directly applied to any solvable BSE a three-dimensional equation without the correct one-body limit is found. However, as demonstrated by Wallace [18], and recapitulated here in Section [V], a Bethe-Salpeter equation with the correct one-body limit may be reorganized so that the pieces of the kernel which contribute at leading order in the one-body limit take on an iterative form. Hence a four-dimensional equation, Eq. (4.17), with the correct one-body limit and meson-production thresholds is derived. A further approximation leads to a simple four-dimensional equation to which the procedure of Section $\llbracket$ may be applied, Eq. (4.26). In making that approximation some of the meson-production thresholds are displaced. Nevertheless, the equation still has exactly the correct cut-structure for $m_{2}-m_{1}<E<m_{1}+m_{2}+\mu$. 
In Section $\square$ the procedure of Section $\llbracket$ was applied to the four-dimensional equation (4.26). The result is a simple three-dimensional equation with the correct one-body limit which has straightforward boost properties. The main shortcoming of this equation is the fact that certain thresholds of the original Bethe-Salpeter wave function are modified in the three-dimensional wave function. However, this modification is due to the approximation in moving from (4.17) to (4.26), rather than to any deficiencies of our procedure for deriving three-dimensional equations. It could be systematically corrected for, at the price of complicating the three-dimensional kernel.

Finally, in Section VI we compared and contrasted the bound-state properties predicted by six different bound-state equations in a scalar field theory. A first group of equations contained the ladder Bethe-Salpeter equation and two three-dimensional equations based on it: the Salpeter equation, and the equation obtained by applying our method to first order - which we referred to as the Klein equation, since the formula obtained for the threedimensional interaction appears in Klein's work. In the second group were Eq. (4.26), the so-called equal-time equation and the equation derived in Section VI. It was found that in each group of three equations the equation derived by the Klein-like delta-function-free reduction technique of Section II approximated its parent four-dimensional equation better than the corresponding instant equation did. However, the bound-state spectra from the instant ET equation lies closest to the bound-state spectrum obtained from the sum of ladder and crossed-ladder graphs, as calculated by Nieuwenhuis and Tjon 35 38. This can be understood on physical grounds and, we argued, is not necessarily a recommendation for the use of the ET equation in physical systems. Indeed, when the wave functions of a two-body system in a scalar field theory are examined it is seen that the equation derived in Section $\mathrm{V}$ does a much better job of reproducing the integrated four-dimensional wave function than the ET equation wave function does.

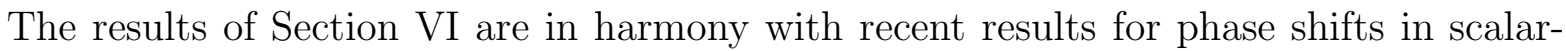
scalar scattering obtained by Lahiff and Afnan [34]. They found that the Klein method reproduces the phase shifts obtained from the corresponding Bethe-Salpeter equation much better than a Blankenbecler-Sugar calculation with an instant interaction.

These formal and numerical results indicate that the procedure of Section $\mathbb{\square}$ is successful in its goal of providing a way to systematically obtain three-dimensional equations from four-dimensional ones without the use of delta functions. In particular, when applied to the four-dimensional equation (4.26) this procedure yields a three-dimensional equation which, unlike the corresponding quasi-potential equation (5.5), has a well-defined boost. This would seem to make such an equation a good starting point for calculations in few-hadron systems.

\section{ACKNOWLEDGMENTS}

S. J. W. acknowledges a conversation in which Mr. Paul Dulany suggested that deltafunction constraints were unnecessary in the three-body problem and a three-dimensional reduction of the Bethe-Salpeter amplitude could be effected by integrating out relative energies. This idea stimulated the present work on the two-body problem. D. R. P. thanks Coen van Antwerpen for his help in writing the code used to solve the Bethe-Salpeter equation. We are grateful to the U.S. Department of Energy for its support under contract no. DE-FG02-93ER-40762. 


\section{REFERENCES}

[1] E. E. Salpeter and H. A. Bethe, Phys. Rev. 84, 1232 (1951).

[2] M. Gell-Mann and F. E. Low, Phys. Rev. 84, 350 (1951).

[3] Y. Nambu, Prog. Theor. Phys. 5, 614 (1950).

[4] J. Schwinger, Proc. Natl. Acad. Sci. U. S. 37, 452 (1951).

[5] J. Schwinger, Proc. Natl. Acad. Sci. U. S. 37, 455 (1951).

[6] M. J. Levine, J. Wright, and J. A. Tjon, Phys. Rev. 154, 1433 (1967).

[7] H. Ito et al., Prog. Theor. Phys. 37, 372 (1967).

[8] J. Fleischer and J. A. Tjon, Nucl. Phys. B84, 375 (1975).

[9] J. Fleischer and J. A. Tjon, Phys. Rev. D 15, 2537 (1977).

[10] N. Nakanishi, Suppl. Prog. Theor. Phys. 43, 1 (1969).

[11] F. Gross, Phys. Rev. C 26, 2203 (1982).

[12] R. Blankenbecler and R. Sugar, Phys. Rev. 142, 1051 (1966).

[13] F. Gross, Phys. Rev. 186, 1448 (1969).

[14] F. Gross, J. W. van Orden, and K. Holinde, Phys. Rev. C 45, 2094 (1992).

[15] E. Hummel and J. A. Tjon, Phys. Rev. C 49, 21 (1994).

[16] J. W. van Orden, N. K. Devine, and F. Gross, Phys. Rev. Lett. 75, 4369 (1995).

[17] V. B. Mandelzweig and S. J. Wallace, Phys. Lett. B. 197, 469 (1987).

[18] S. J. Wallace, in Nuclear and Particle Physics on the Light Cone, edited by M. B. Johnson and L. S. Kisslinger (World Scientific, Singapore, 1988).

[19] S. J. Wallace and V. B. Mandelzweig, Nucl. Phys. A503, 673 (1989).

[20] N. K. Devine and S. J. Wallace, Phys. Rev. C 48, 973 (1993).

[21] N. K. Devine and S. J. Wallace, Phys. Rev. C 51, 3222 (1995).

[22] M. Fuda, Ann. Phys. (N.Y.) 197, 265 (1990).

[23] A. Klein, Phys. Rev. 90, 1101 (1953).

[24] A. Klein, Phys. Rev. 94, 1052 (1954).

[25] A. Klein and B. H. McCormick, Prog. Theor. Phys. 20, 876 (1958).

[26] A. Klein and T.-S. H. Lee, Phys. Rev. D 10, 4308 (1974).

[27] J. L. Forest, V. R. Pandharipande, and J. L. Friar, Phys. Rev. C 52, 568 (1995).

[28] A. N. Kvinikhidze and B. Blankleider, Few-Body Systems, Suppl. 7, 294 (1994).

[29] A. A. Logunov and A. N. Tavkhelidze, Nuovo Cim. 29, 380 (1963).

[30] E. E. Salpeter, Phys. Rev. 87, 328 (1952).

[31] D. R. Phillips, Ph.D. thesis, Flinders University of South Australia, 1995.

[32] T. A. Rijken, Ann. Phys. (N.Y.) 208, 253 (1991).

[33] T. A. Rijken and V. G. J. Stoks, Nuclear Theory preprint archive nos. nucl-th/9509029 and nucl-th/9509031, submitted to Phys. Rev. C.

[34] A. D. Lahiff and I. R. Afnan, in preparation.

[35] J. A. Tjon and Y. A. Simonov, Ann. Phys. (N.Y.) 228, 1 (1993).

[36] T. Nieuwenhuis, J. A. Tjon, and Y. A. Simonov, Few Body Systems Suppl. 7, 286 (1994).

[37] T. Nieuwenhuis, Ph.D. thesis, University of Utrecht, 1995.

[38] T. Nieuwenhuis and J. A. Tjon, in preparation.

[39] T. Nieuwenhuis and J. A. Tjon, Phys. Lett. B. 355, 283 (1995).

[40] E. Cooper and B. K. Jennings, Nucl. Phys. A500, 553 (1989).

[41] P. Tiemeijer and J. A. Tjon, Phys. Lett. B. 277, 38 (1992). 
[42] P. Tiemeijer and J. A. Tjon, Phys. Rev. C 48, 896 (1993). 


\section{FIGURES}

FIG. 1. The two graphs which contribute to the second-order three-dimensional kernel, if only positive-energy intermediate states are included in the calculation.

FIG. 2. The ten graphs which contribute to the second-order three-dimensional kernel if propagation into negative-energy states is allowed. Note that these graphs can be interpreted as applying to positive or negative-energy particles. In fact, each graph shown contributes twice: the second time with positive-energy particles going forward in time interchanged with negative-energy particles going backward. The dotted lines represent the initial and final times in each graph. Observe that in all but graphs (a) and (e) some interactions take place outside the interval $\left[t_{i}, t_{f}\right]$.

FIG. 3. The crossed-box graph, showing the momentum labels used in the text.

FIG. 4. A plot of bound-state mass $\left(\frac{M}{m}\right)$ versus coupling strength $\left(\frac{g_{1} g_{2}}{\pi}\right)$ for the seven different calculations discussed in the text. The points with error bars are taken from [37] and represent the FSR calculation of Nieuwenhuis and Tjon. The other calculations were all performed using three or four-dimensional integral equations, with the legend as indicated in the figure.

FIG. 5. A plot of normalized wave-functions $k \psi(k)$ versus momentum $k$ (in units such that $m=1$ ), for a bound-state with $M=1.95 \mathrm{~m}$. The different wave functions shown are the integrated ladder BSE wave function (solid line); the result of the Klein potential calculation (long dashed line) and the Salpeter equation wave function (dotted line).

FIG. 6. A plot of normalized wave-functions $k \psi(k)$ versus momentum $k$ (in units such that $m=1$ ), for a bound-state with $M=1.95 \mathrm{~m}$. The different wave functions shown are the integrated four-dimensional Wallace-Mandelzweig equation wave function (thick solid line); the wave-function resulting from the first-order relative-energy integration method applied to that equation (dash-dotted line) and the ET wave function (short dashed line). 

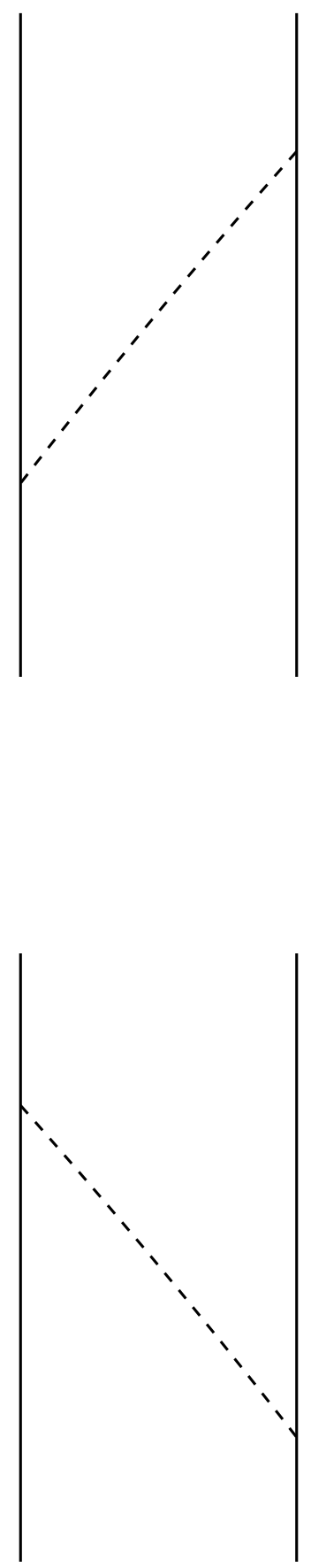


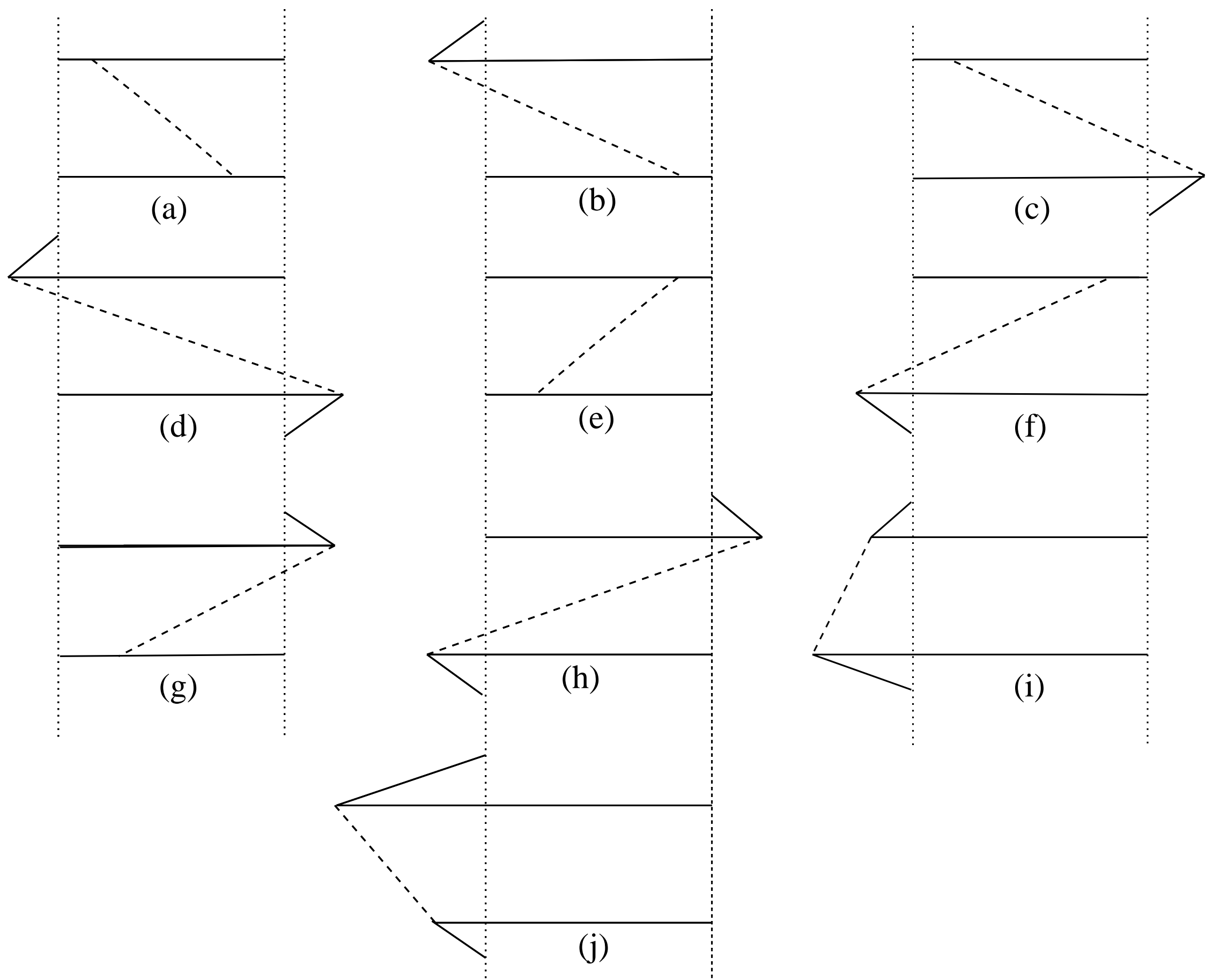




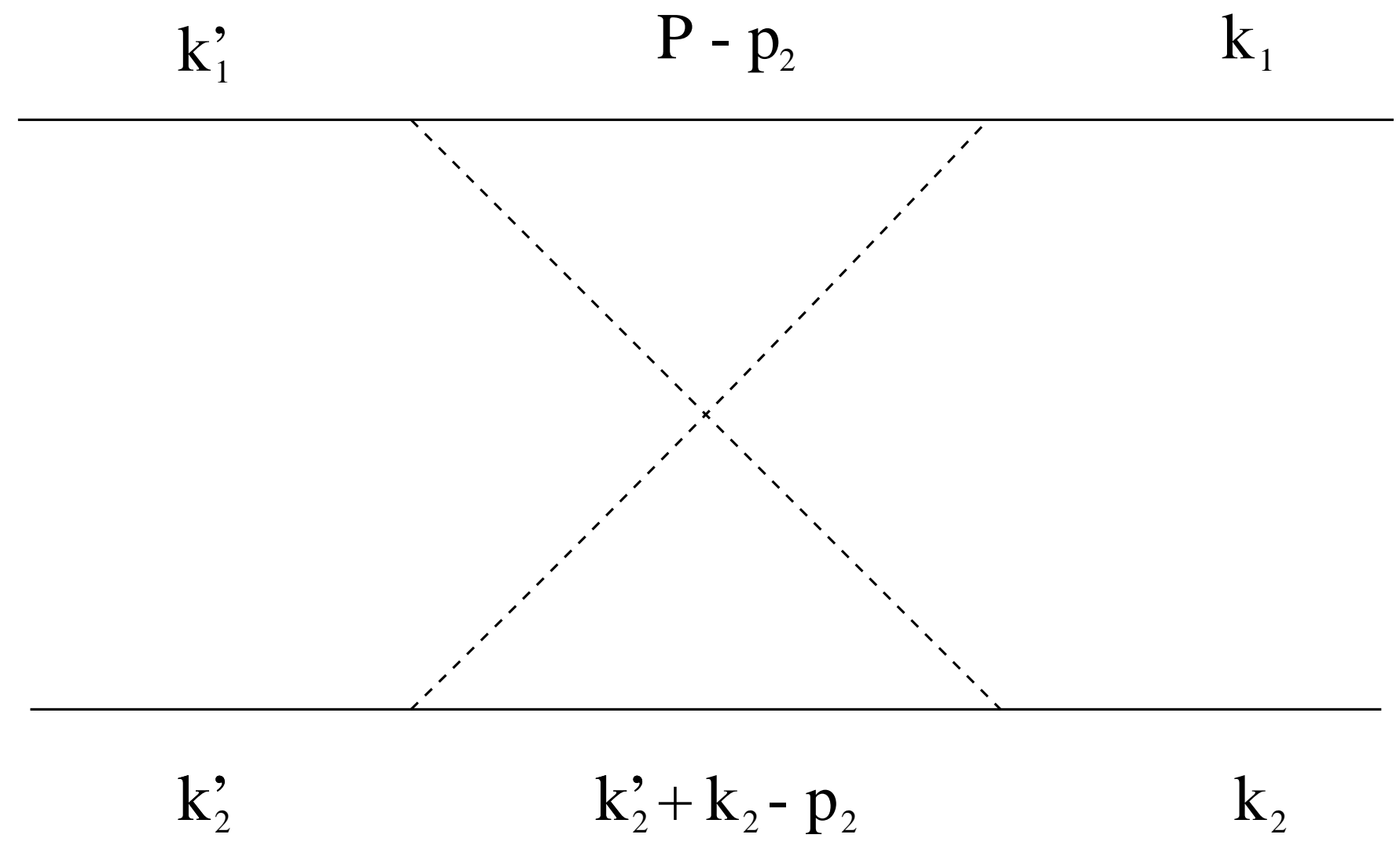




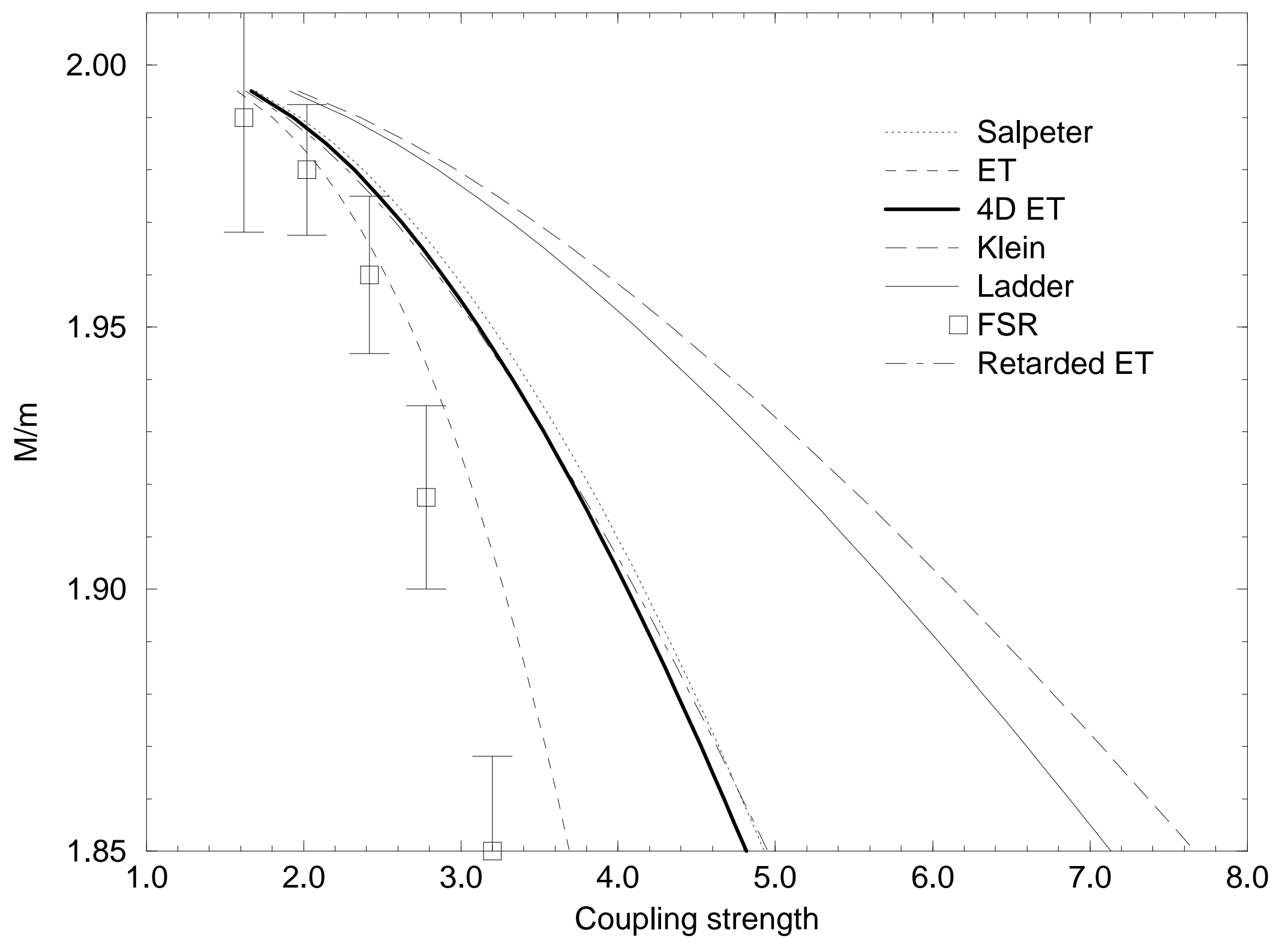




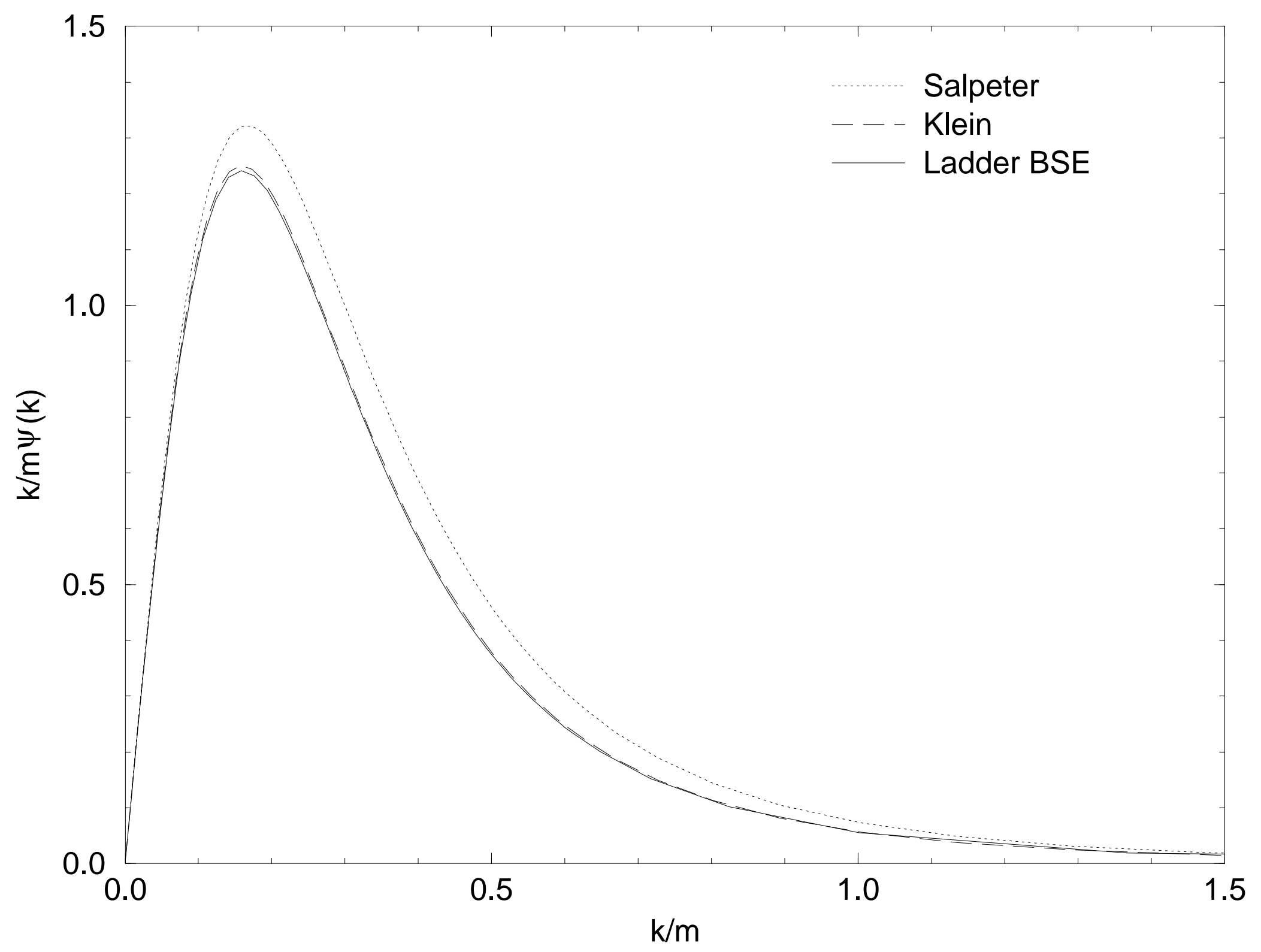




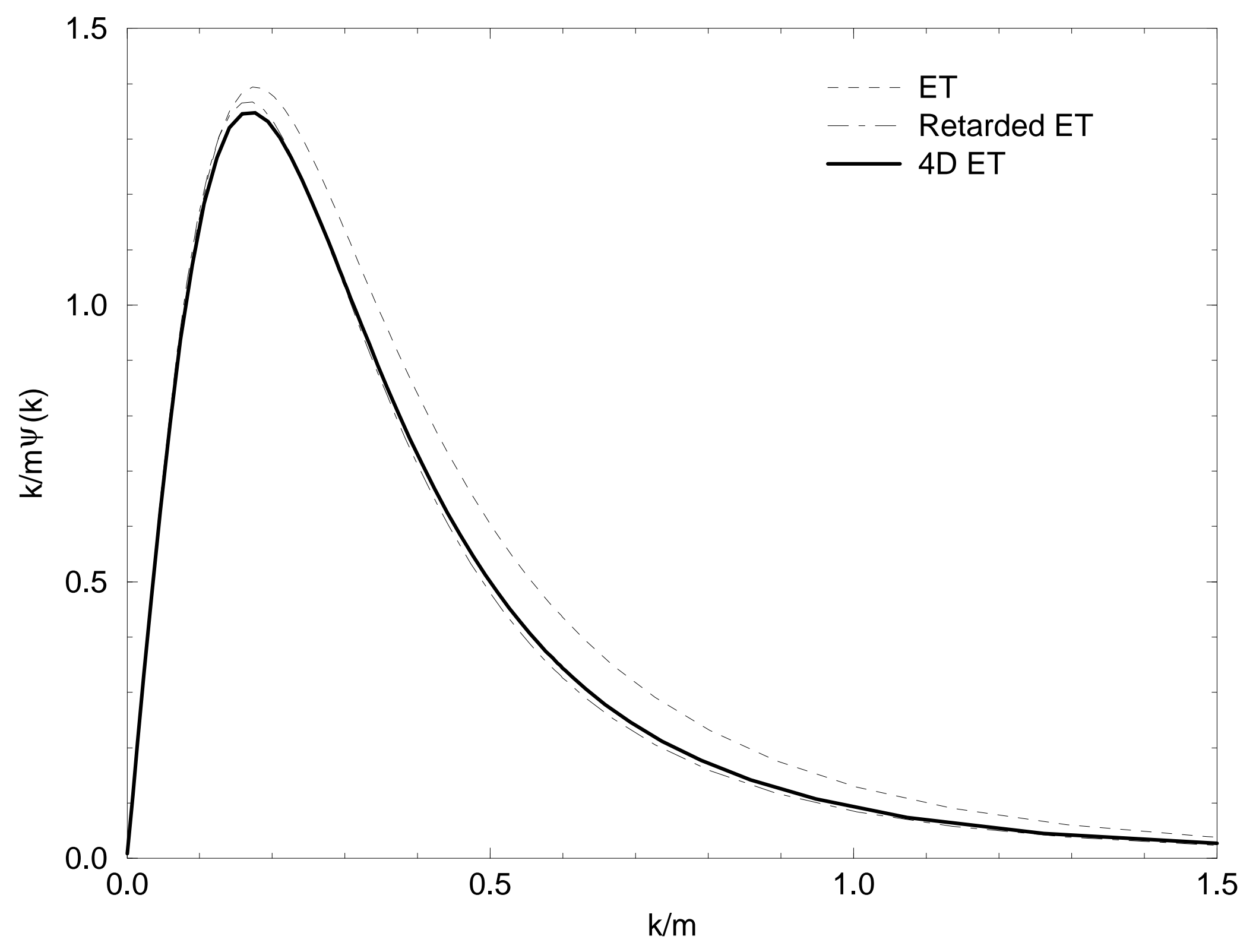

